\title{
Symmetries and conservation laws in quantum trajectories: Dissipative freezing
}

\author{
Carlos Sánchez Muñoz $\odot,{ }^{1, *}$ Berislav Buča, ${ }^{1}$ Joseph Tindall $\odot,{ }^{1}$ Alejandro González-Tudela, ${ }^{2}$ \\ Dieter Jaksch ${ }^{10},{ }^{1}$ and Diego Porras ${ }^{2}$ \\ ${ }^{1}$ Clarendon Laboratory, University of Oxford, Parks Road, Oxford OX1 3PU, United Kingdom \\ ${ }^{2}$ Instituto de Física Fundamental, CSIC, Calle Serrano 113b, Madrid 28006, Spain
}

(Received 15 July 2019; published 16 October 2019)

\begin{abstract}
In driven-dissipative systems, the presence of a strong symmetry guarantees the existence of several steady states belonging to different symmetry sectors. Here we show that when a system with a strong symmetry is initialized in a quantum superposition involving several of these sectors, each individual stochastic trajectory will randomly select a single one of them and remain there for the rest of the evolution. Since a strong symmetry implies a conservation law for the corresponding symmetry operator on the ensemble level, this selection of a single sector from an initial superposition entails a breakdown of this conservation law at the level of individual realizations. Given that such a superposition is impossible in a classical stochastic trajectory, this is a purely quantum effect with no classical analog. Our results show that a system with a closed Liouvillian gap may exhibit, when monitored over a single run of an experiment, a behavior completely opposite to the usual notion of dynamical phase coexistence and intermittency, which are typically considered hallmarks of a dissipative phase transition. We discuss our results on a coherently driven spin ensemble with a squeezed superradiant decay, a simple model that presents a wealth of nonergodic dynamics.
\end{abstract}

DOI: 10.1103/PhysRevA.100.042113

\section{INTRODUCTION}

Driven-dissipative systems are ubiquitous in many-body physics and cavity QED [1-12]. These systems are typically gapped and feature a unique nonequilibrium steady state. In the regime of a dissipative phase transition (DPT), however, this gap vanishes and the null space of the Liouvillian is spanned by several compatible steady states [13-23]. Due to their fundamental interest and practical applications, such as enhanced metrological properties [24,25], DPTs have attracted a significant amount of attention, with much work being devoted to studying the associated phenomena of bistability [3-5,22,26-29], hysteresis [2,30], intermittency [6,27,3033], multimodality [26,32], metastability [34], and symmetry breaking [35-37]. All these effects are understood as different manifestations of the coexistence of several nonequilibrium phases. In particular, many experiments will look for intermittency as the hallmark of such phase coexistence $[6,27,30-33]$. Intermittency is a phenomenon defined by a random switching between periods of high and low dynamical activity (for instance, in the rate of photon emission). This behavior, which is observed during a single run of the experiment, is conveniently described using the formalism of quantum jumps in which the system is characterized in terms of a pure wave function that undergoes stochastic evolution [38-40].

The timescale $\tau$ of this intermittency is given by the inverse of the Liouvillian gap or asymptotic decay rate (ADR), i.e., the eigenvalue $\lambda_{2}$ of the Liouvillian operator $\mathcal{L}$ with the second largest real part $[24,41,42]$. Since a DPT is defined

\footnotetext{
*carlos.sanchezmunoz@physics.ox.ac.uk
}

by a vanishing Liouvillian gap $[13,14]$, it will necessarily imply that $\tau$ diverges. In most typical situations, this closing is reached in the thermodynamic limit of a many-body system. Consequently, for any finite system, the long-time limit where intermittency is observable will, at least formally, exist. There are however situations in which the Liouvillian gap vanishes exactly and such a long-time limit cannot be taken. This is the case of systems featuring a strong symmetry [43]. Liouvillians $\mathcal{L}$ with a strong symmetry have a degenerate steady state (implying that $\lambda_{2}=0$ ) and an associated conservation law for the symmetry operator $\dot{A}=\mathcal{L}^{\dagger} A=0$ [43,44]. Since the Liouvillian gap is closed exactly for any system size, the long-time limit of intermittency described before does not exist and the dynamics is split into different, unconnected ergodic symmetry sectors.

In this work we study the quantum trajectories of open quantum systems with a strong symmetry. We show that when initialized in a superposition involving different symmetry sectors, the system will evolve towards a single one of them in each individual trajectory, remaining there for the rest of the realization. This nonergodic phenomenon, which we term dissipative freezing, is in stark contrast with the typically sought phenomenology of intermittency in a DPT and predicts a completely different dynamical behavior at the level of individual realizations of the experiment. Related effects have already been discussed in different contexts: In Ref. [45] exponential stability of subspaces for quantum trajectories was demonstrated, in Ref. [46] it was shown that a quantum stochastic master equation describing nondemolition measurements converges to a pure state, and in Ref. [47] a similar effect was discussed for quantum Markov chains. An important result of our work is to relate this phenomenon to the symmetries of the master equation. Notably, this implies that the 
conservation law for the strong-symmetry operator is broken at the level of trajectories and can only be recovered under ensemble averaging. This is a purely quantum phenomenon, since it requires an initial superposition of different symmetry sectors that cannot be implemented classically, i.e., a single classical trajectory is always fully realistic and located in only one of this sectors. Understanding this phenomenon is important for the dynamical characterization of dissipative systems with a closed Liouvillian gap. This limit has been proven relevant in quantum metrology, since it yields a Heisenberg scaling (quadratic in time) of the quantum Fisher information [24].

To introduce and discuss the phenomenon of dissipative freezing, we analyze a model that can be solved numerically yet displays a rich variety of nonergodic dynamics. This model consists of a coherently driven spin ensemble with squeezed collective spin decay, which can be implemented by adiabatic elimination of a cavity mode coupled to a multicomponent atomic condensate via cavity-assisted Raman transitions [48-52]. Squeezed decay refers to a quantum-jump operator that includes both lowering and raising collective spin operators $S_{ \pm}$, with relative weights parametrized by a squeezing angle $\theta$. The amplitude $\Omega$ of the driving field and the squeezing angle $\theta$ are the main tunable parameters, which allow one to define a nonequilibrium phase diagram in the $(\Omega, \theta)$ plane.

While this model is simple enough to be treated numerically, it displays a variety of dissipative phases with a gapless Liouvillian. The characterization of these types of models is highly relevant, since one of the problems that hinder our understanding of nonequilibrium systems and DPTs is the enormous computational difficulty typically found when dealing with large quantum open systems. It is thus desirable to work with exactly solvable systems or at least computationally tractable models that yield insight into the physics in the thermodynamic limit.

The nonequilibrium gapless phases of this model are associated with different nonergodic behaviors that depart from the usual pictures of phase coexistence. Beyond the case of dissipative freezing, which is the main focus of this work, the second nonergodic phenomenon observed in this model is the appearance of oscillatory nonstationary dynamics in the long-time limit. This effect is related to the existence of a spectrum of purely imaginary eigenvalues of $\mathcal{L}$, which needs to be equally spaced in order to prevent eigenstate thermalization. This phenomenon has recently attracted attention in similar systems [53-55] and has been linked to the existence of a dynamical symmetry in the system [53,56], e.g., a ladder operator of the Hamiltonian that commutes with all the quantum-jump operators of the Liouvillian.

The paper is organized as follows. In Sec. II we introduce the model of squeezed superradiance, describing the phase diagram and steady state of the system. In Sec. III we analyze the Liouvillian spectrum of this model and characterize symmetries and regimes of nonergodicity. In Sec. IV we describe the phenomenon of dissipative freezing and discuss it in the context of thermodynamics of quantum trajectories and phase transitions. In Sec. V we analyze the signatures of critical dissipative dynamics in observables of the light emitted by the system. We summarize in Sec. VI.

\section{MODEL AND PHASE DIAGRAM}

\section{A. Squeezed superradiance: Derivation of the spin master equation}

The model of squeezed superradiance that we consider in this work is given by the master equation for the reduced density matrix of an ensemble of $N$ spins $(\hbar=1)$

$$
\dot{\rho}=-i \Omega\left[S_{x}, \rho\right]+\frac{\Gamma}{2 J} \mathcal{L}_{D_{\theta}}[\rho],
$$

where $\mathcal{L}_{\mathcal{O}}[\rho] \equiv 2 \mathcal{O} \rho \mathcal{O}^{\dagger}-\left\{\mathcal{O}^{\dagger} \mathcal{O}, \rho\right\}$ is the usual Lindblad superoperator and the operator $D_{\theta}$ describes the quantum jumps undergone by the system

$$
D_{\theta} \equiv \cos (\theta) S_{-}+\sin (\theta) S_{+} .
$$

In these equations, $\left\{S_{ \pm}, S_{z}\right\}$ are collective spin operators obeying angular momentum commutation relations, $\Omega$ is the driving amplitude, $\Gamma$ is the quantum-jump rate, and $J=N / 2$ is the total angular momentum which is conserved in the dynamics. Notably, $D_{\theta}$ includes both raising and lowering operators, with a weight that we parametrize by the angle $\theta$.

The dynamics in Eq. (1) emerge as the strongly dissipative limit of the Hamiltonian

$$
H=\Omega S_{x}+\frac{g}{\sqrt{N}}\left\{S_{+}\left[\cos (\theta) a+\sin (\theta) a^{\dagger}\right]+\text { H.c. }\right\} .
$$

This Hamiltonian describes a driven spin ensemble coupled to a single cavity mode in the rotating frame of the driving, with $a$ the bosonic annihilation operator of the cavity and $g$ the spin-cavity coupling rate. Since the total angular momentum $J$ is conserved, the spin ensemble can be described as a single big spin; this can be implemented, for instance, with multicomponent atomic condensates [48,49]. The tunable coupling terms in Eq. (3) can be achieved via cavity-assisted Raman transitions; this approach has been proposed as a way to implement effective Dicke models [50] and used successfully to observe alternative forms of the superradiant phase transition [57-60] in atomic condensates $[7,8,61,62]$ and thermal atoms $[63,64]$. The great control and versatility provided by these schemes has motivated research on generalized nonequilibrium Dicke models [65,66].

In this work we are focusing on strongly dissipative versions of these systems (where the fast cavity decay yields an effective collective spin dissipation), which have attracted interest for their applications to the dissipative generation of spin squeezing and entanglement in the steady state [51,52]. By taking into account that the cavity experiences dissipation at a rate $\gamma$, the evolution of the system is described by the master equation [67] $\dot{\rho}=-i[H, \rho]+\gamma / 2 \mathcal{L}_{a}[\rho]$. In the limit $\gamma \rightarrow$ $\infty$, the bosonic field tends to a stationary vacuum state, and its adiabatic elimination [52] yields the effective dynamics for the spins of Eq. (1), with $\Gamma=2 g^{2} / \gamma$. It is easy to deduce that the dark state of $D_{\theta}$ is a spin squeezed state [51], which leads us to refer to the dissipative part of Eq. (1) as a squeezed decay. Note that, when $\theta=0$, the model corresponds to the standard case of collective resonance fluorescence [52,68,69].

\section{B. Phase diagram}

The nonequilibrium phases of the system in the $(\Omega, \theta)$ plane are summarized in Fig. 1(a) and the corresponding 
(a)

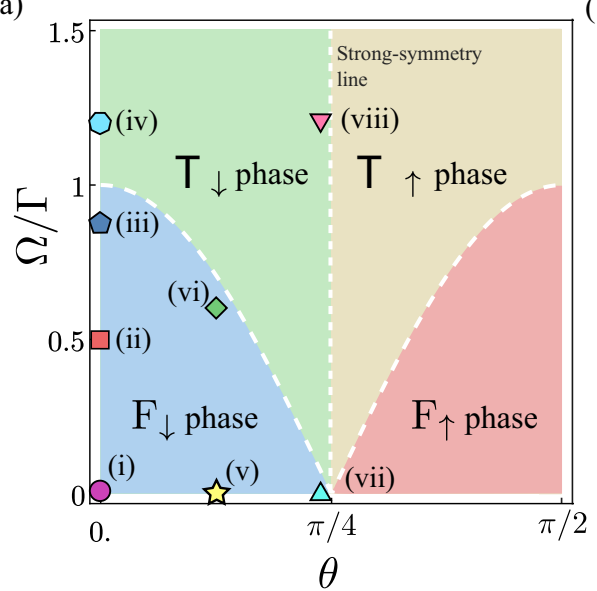

(b)

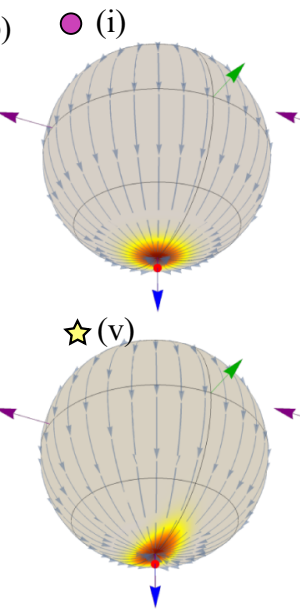

(ii)

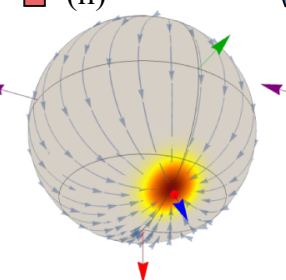

(iii)

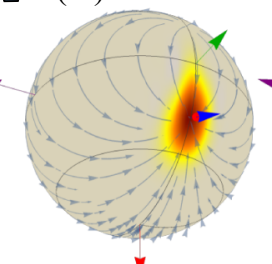

(iv)

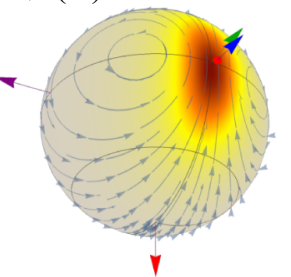

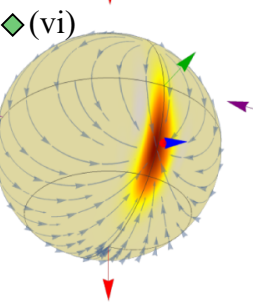
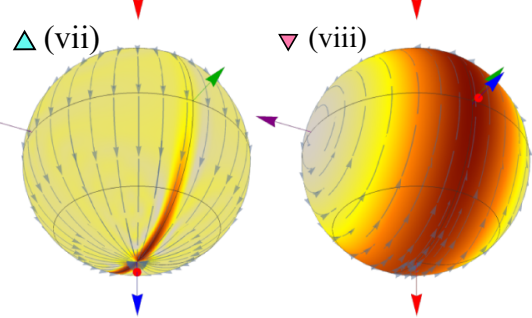

FIG. 1. Phase diagram and steady state. (a) The phase diagram can be divided into a ferromagnetic (F) and a thermal (T) phase, separated by the critical line $\Omega_{c}(\theta)$ given by Eq. (5) (white dashed lines). There is a spin-up and a spin-down version of each of these phases, separated by the strong-symmetry line $\theta=\pi / 4$. (b) Spin Wigner functions of the exact steady states of the master equation (1) for a finite system with $N=50$ at different points $(\Omega, \theta)$, corresponding to (i) $(0,0)$, (ii) $(0.5 \Gamma, 0)$, (iii) $(0.88 \Gamma, 0)$, (iv) $(1.2 \Gamma, 0),(\mathrm{v})(0, \pi / 8),(\mathrm{vi})(0.6 \Gamma, \pi / 8)$, (vii) $(0,0.95 \pi / 4)$, and (viii) $(1.2 \Gamma, 0.95 \pi / 4)$. Together we plot the vector field of the derivatives described by the mean-field equations $(\mathrm{A} 3)$.

steady-state observables computed exactly for a finite system $(N=50)$ are depicted in Fig. 2. Figure 1(b) depicts the steady state of a finite system $(N=50)$ at several points of the phase diagram using the spin Wigner function [70,71]. Additionally, we plot the vector field of derivatives obtained through a mean-field approach (see Appendix A). We can divide the phase diagram into two types of phases.

(i) The ferromagnetic phase is characterized by a welldefined magnetization [cf. Fig. 2(a)], a diverging spin squeezing as we approach the phase transition [Fig. 2(b)], small fluctuations in the counting distributions of quantum jumps (described here by the zero-delay second-order correlation function of the output field $\left.g^{(2)} \equiv\left\langle D_{\theta}^{\dagger^{2}} D_{\theta}{ }^{2}\right\rangle /\left\langle D_{\theta}^{\dagger} D_{\theta}\right\rangle^{2}\right)$ [Fig. 2(d)], high purity (not shown), and ergodic dynamics. Any initial state eventually relaxes into a stationary, highly pure Gaussian steady state. In the thermodynamic limit, this phase is well described within a Holstein-Primakoff approximation.

(ii) In the thermal phase the steady state is highly mixed, and close to the infinite-temperature state $\rho \propto \mathbb{1}$. This phase is characterized by a mean zero magnetization [Fig. 2(a)], small purity (not shown), large spin fluctuations, high rate of quantum jumps (activity) [Fig. 2(c)], and large fluctuations in the output field [Fig. 2(d)]. As we discuss further below, this phase displays a vanishing ADR that leads, in the thermodynamic limit $N \rightarrow \infty$, to a closed gap and nonergodic dynamics, which manifests itself through closed orbits in the mean-field approach [cf. point (iv) in Fig. 1(b)].

Both phases have a spin-down $(\downarrow)$ and spin-up $(\uparrow)$ version at each side of the line $\theta=\pi / 4$, each of them being a spinflipped version of the other. Therefore, defining

$$
\begin{aligned}
& \Gamma_{-} \equiv \Gamma \cos ^{2} \theta, \\
& \Gamma_{+} \equiv \Gamma \sin ^{2} \theta,
\end{aligned}
$$

all the results and equations obtained for $\theta \leqslant \pi / 4$ are directly applicable in a spin-flipped basis for $\theta \geqslant \pi / 4$ just by exchanging $\Gamma_{-} \leftrightarrow \Gamma_{+}$. Hereafter, the analytical results that we provide refer to the spin-down phases $(\theta \leqslant \pi / 4)$. In Appendix A we show that, using a mean-field approach, the transition from the ferromagnetic to the thermal phase occurs at the critical driving

$$
\Omega_{c}(\theta)=\Gamma_{-}-\Gamma_{+}=\Gamma\left(\cos ^{2} \theta-\sin ^{2} \theta\right) .
$$

\section{Spin observables}

We consider now the expectation values of the normalized spin operators $s_{i} \equiv S_{i} / J, i \in\{x, y, z\}$, in the steady state. In the ferromagnetic phase, these can be obtained by a displaced Holstein-Primakoff (HP) expansion (see Appendix B); the results are the same as the mean-field predictions, with corrections to order $1 / J$,

$$
\begin{aligned}
& \left\langle s_{z}\right\rangle=M+O(1 / J), \\
& \left\langle s_{x}\right\rangle=0+O(1 / J), \\
& \left\langle s_{y}\right\rangle=\sqrt{1-M^{2}}+O(1 / J),
\end{aligned}
$$

where $M$ is the steady-state magnetization that reads

$$
M=-\sqrt{1-\left(\frac{\Omega}{\Gamma_{-}-\Gamma_{+}}\right)^{2}} .
$$

The $1 / J$ corrections are given by the solution of nonquadratic master equations and therefore analytical expressions are difficult to obtain. It is however possible to get expressions for the spin fluctuations $\Delta s_{z / \pm}^{2}$ to order $1 / J$; this is one of the main advantages of using a HP expansion, since it allows one to describe the metrological properties of the spin ensemble [72]. In particular, reduced fluctuations along one of the spin directions provides enhanced phase sensitivity in atomic interferometers $[73,74]$ and greater stability in atomic clocks [75]. States displaying such reduced fluctuations are said to be spin squeezed [72,76,77]; the degree of spin squeezing $\xi_{\perp}$ along any axis $\mathbf{u}_{\perp}$ perpendicular to the mean spin direction is a popular figure of merit, useful as a witness of entanglement [78] and as a direct measure of the 

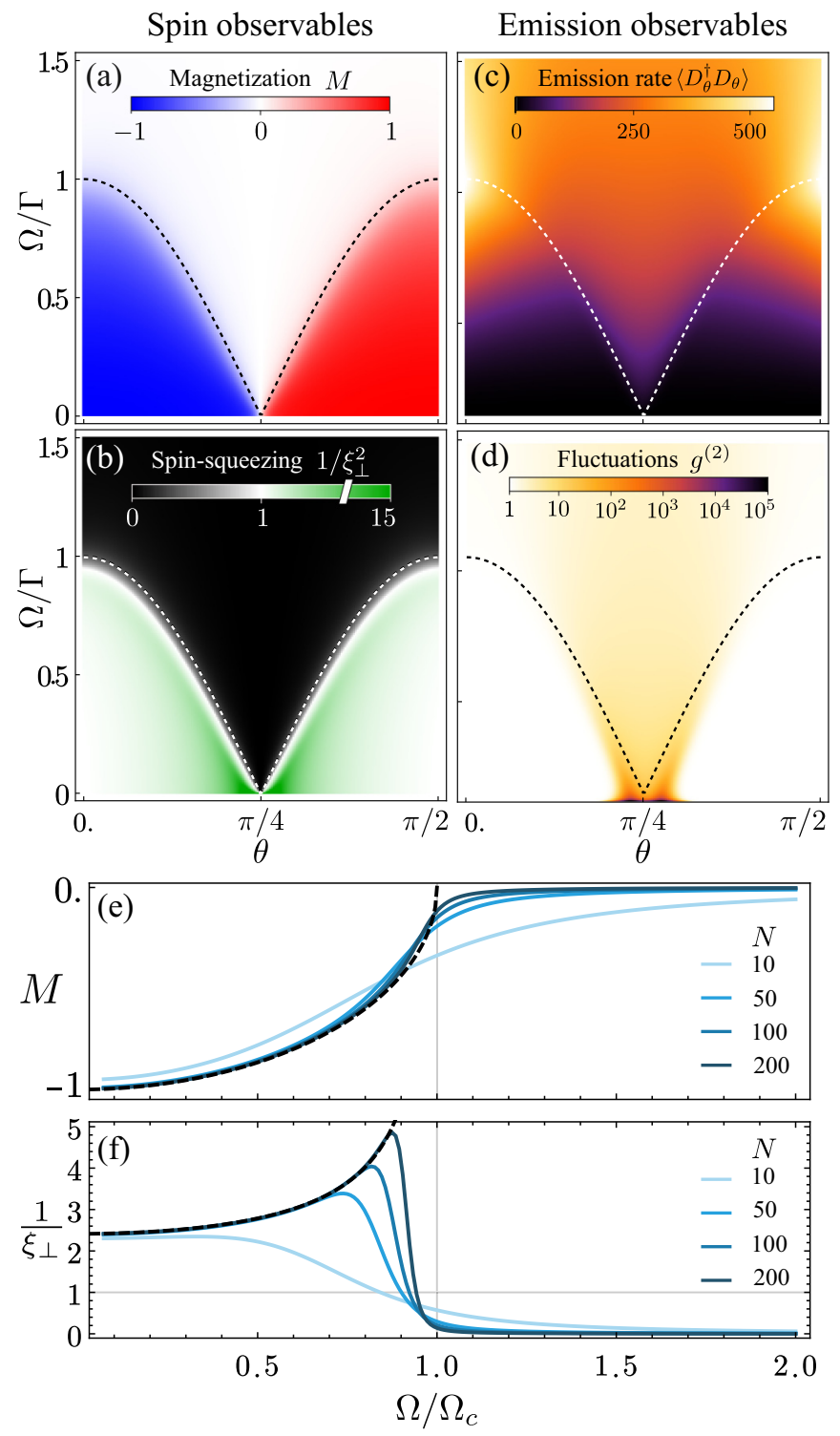

FIG. 2. (a)-(d) Steady-state observables for a finite system size $N=50$. Dashed lines indicate the critical line [Eq. (5)]. Also shown is the (e) magnetization and (f) degree of spin squeezing across the phase transition. The black dashed line is the analytical value in the thermodynamic limit [Eqs. (7) and (9)]. Solid lines are numerical calculations for finite systems of different sizes. Calculations were made at $\theta=\pi / 8$.

phase sensitivity achievable in interferometry protocols. This quantity can be defined as [76]

$$
\xi_{\perp}^{2}=\frac{N\left(\Delta S_{\perp}\right)^{2}}{\langle\mathbf{S}\rangle^{2}} .
$$

According to this definition, a state is spin squeezed if a direction $\mathbf{u}_{\perp}$ exists such that $\xi_{\perp}^{2}<1$. In our model, the optimal squeezing direction is always the $\mathbf{u}_{\mathbf{x}}$ axis (see Appendix B 4). Using the HP approximation, we find the following expression for the spin squeezing in the ferromagnetic phase:

$$
\xi_{\perp}^{2}=\frac{N\left(\Delta S_{x}\right)^{2}}{\langle\mathbf{S}\rangle^{2}}=(1-M)\left(\frac{1}{2}+\frac{\Gamma_{+}-\sqrt{\Gamma_{-} \Gamma_{+}}}{\Gamma_{-}-\Gamma_{+}}\right) .
$$

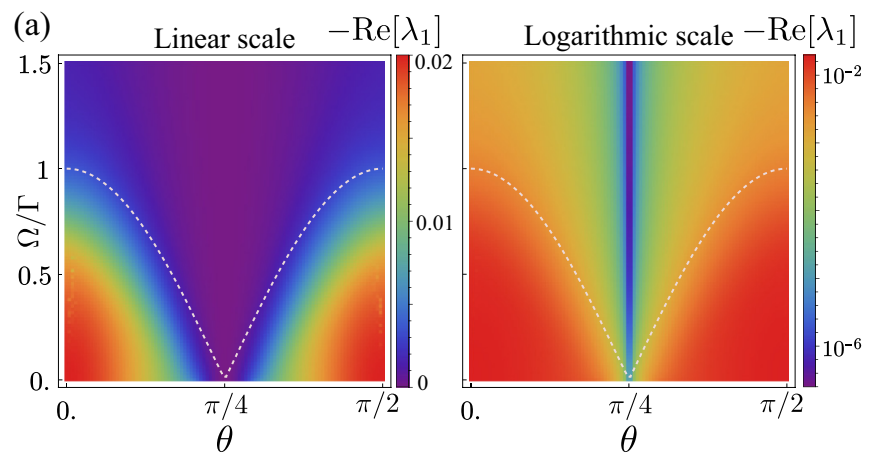

(b)

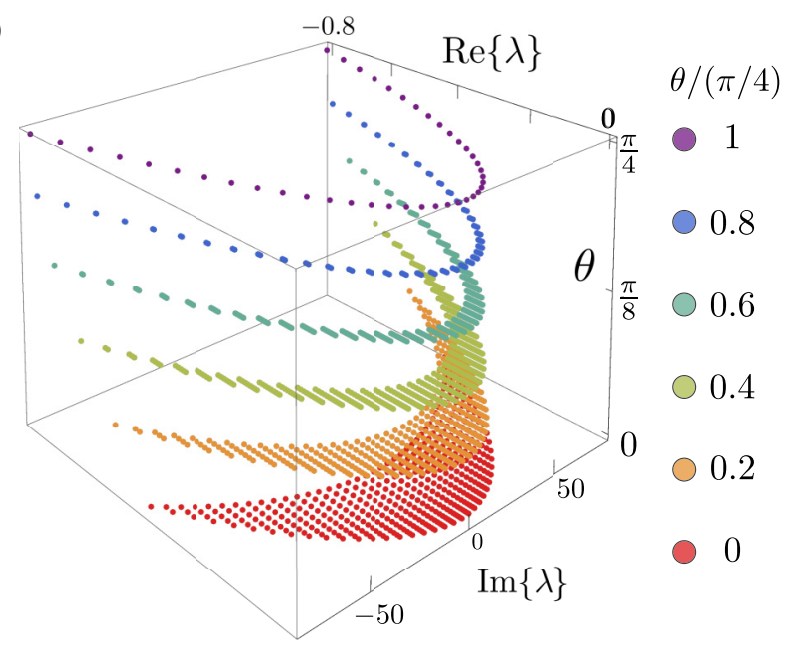

FIG. 3. (a) Liouvillian gap for $N=100$ and $\Omega=0.4 \Gamma$. In the thermodynamic limit, the gap closes at the critical line $\Omega_{c}(\theta)$ (white dashed line). In logarithmic scale, we observe a closing of the gap for finite $J$ at the point $\theta=\pi / 4$ due to the strong symmetry. (b) Liouvillian eigenvalues for a system size $J=10$ and $\Omega=200 \Gamma$.

The analytical results in Eqs. (6) and (9) are shown in Figs. 2(e) and 2(f), compared with numerical calculations for finite system size. Equation (9) shows that, in the thermodynamic limit, spin squeezing diverges (i.e., $\xi_{\perp}^{2} \rightarrow 0$ ) in the vicinity of the critical line, where $M \rightarrow 0$. This implies a greatly enhanced phase sensitivity and the emergence of many-body correlations, which are general properties associated with second-order phase transitions [13].

\section{SPECTRAL PROPERTIES OF THE LIOUVILLIAN}

Having characterized the phase diagram of the model, we analyze now the spectral properties of the Liouvillian, which contains essential information about the different dissipative phases and nonergodic dynamics $[13,14,34]$. In the ferromagnetic phase, we can use the Holstein-Primakoff expansion to obtain an expression for the Liouvillian gap in the thermodynamic limit (see Appendix B)

$$
\lambda=\left(\Gamma_{-}-\Gamma_{+}\right) M
$$

showing that the gap closes when $M=0$, i.e., at the transition from a ferromagnetic to a thermal phase, in agreement with the usual description of DPTs [13,14]. Figure 3(a) depicts the exact $\mathrm{ADR}$ for a finite system, computed by numerical 
diagonalization. The ADR in the thermal phase features a small but finite value that, as we prove below, scales with system size as $1 / J$. Below we focus on this gapless region, which is the most promising in terms of nonergodic dynamics.

\section{A. Strong symmetry}

Even for a finite system, the ADR closes exactly at the line that separates the $\mathrm{T}_{\downarrow}$ and $\mathrm{T}_{\uparrow}$ phases, $\theta=\pi / 4$, as can be seen from the logarithmic-scale plot in Fig. 3(a). The reason for this exact closing, which occurs even at finite system size, is the existence of a strong symmetry at $\theta=\pi / 4$. For a general Liouvillian given by $\mathcal{L} \rho=-i[H, \rho]+\sum_{\mu}\left(2 L_{\mu} \rho L_{\mu}^{\dagger}-\right.$ $\left.\left\{L_{\mu}^{\dagger} L_{\mu}, \rho\right\}\right)$, a strong symmetry is defined by a unitary operator $A$ which fulfills

$$
\begin{aligned}
{[H, A] } & =0, \\
{\left[L_{\mu}, A\right] } & =0 .
\end{aligned}
$$

As demonstrated in Ref. [43], the existence of a strong symmetry implies that if $A$ has $n_{A}$ distinct eigenvalues, there are at least $n_{A}$ distinct steady states of $\mathcal{L}$ with eigenvalue 0 . In the particular case $A=H=L$ (with $L \equiv L_{1}$ being the only quantum-jump operator), the density matrices $\rho^{(m)}=$ $|m\rangle\langle m|$ are all steady states, with $|m\rangle$ the eigenstates of $A$. In our system, we find $\{H, L\} \propto S_{x}$ at the strong-symmetry point $\theta=\pi / 4$, which means that $S_{x}$ is a strong symmetry of the Liouvillian and that all its eigenstates are steady states, explaining the exact closing of the ADR. The existence of a strong symmetry at $\theta=\pi / 4$ is key to understanding the effect of dissipative freezing that we discuss in the following section.

\section{B. Imaginary eigenvalues}

A more general analysis of the Liouvillian spectrum in the large driving limit provides further insight into the different ways in which the gap can be closed well within the thermal phase and reveals the existence of eigenstates with purely imaginary values. In the limit $\Omega \gg \Gamma / J$, we can remove counterrotating terms in the master equation and obtain

$$
\dot{\rho} \approx-i \Omega\left[S_{x}, \rho\right]+\frac{\Gamma_{\theta}}{2 J} \mathcal{L}_{S_{x}}[\rho]+\frac{\chi_{\theta}}{8 J}\left(\mathcal{L}_{S_{x}^{+}}[\rho]+\mathcal{L}_{S_{x}^{-}}[\rho]\right),
$$

where we have defined the ladder operators in the $x$ direction, $S_{x}^{ \pm} \equiv \frac{1}{2}\left(S_{z} \pm i S_{y}\right), \Gamma_{\theta} \equiv \Gamma(\cos \theta+\sin \theta)^{2}$, and $\chi_{\theta} \equiv$ $\Gamma(\cos \theta-\sin \theta)^{2}$. For $\theta \neq \pi / 4$, the steady-state solution is the infinite-temperature state $\rho_{\infty}=\mathbb{1} / 2 \mathrm{~J}$. One can find an analytical solution of the eigenvalue problem for this Liouvillian [79]; here we provide compact closed-form expressions for the eigenvalues and relevant eigenstates. The spectrum of eigenvalues reads

$$
\lambda_{q, k}^{ \pm}= \pm i q \Omega-\frac{\Gamma_{\theta}}{2 J} q^{2}-\frac{\chi_{\theta}}{4 J}[q+k(1+k+2 q)],
$$

with $q=0,1, \ldots, 2 J$ and $k=0,1, \ldots, 2 J-q$. This spectrum is plotted in Fig. 3(b) for different values of $\theta$. The corresponding eigenstates can be written in terms of the states

$$
\rho^{(n, m)} \propto\left(S_{x}^{+}\right)^{n} \rho_{\infty}\left(S_{x}^{-}\right)^{m} .
$$

For a given $q$, the $2 J+1-q$ eigenstates corresponding to the eigenvalues $\lambda_{q, k}^{i}$ can be built from superpositions of different $\rho^{(n, m)}$, with $(n, m)$ fulfilling $q=|n-m|$ and $i=\operatorname{sgn}(n-m)$. In particular, the eigenstates with eigenvalue $\lambda_{q, 0}^{ \pm}$, which are the slowest-decaying ones among those having the same $q$ (i.e., same imaginary eigenvalue), take the simple form $\rho^{(q, 0)}$ and $\rho^{(0, q)}$. In the strong-symmetry situation $\theta=\pi / 4$, i.e., when $\chi_{\theta}=0, \rho^{(n, m)}$ are the exact eigenstates themselves.

Equation (13) clearly shows that, besides the eigenvalue $\lambda_{0,0}=0$, which corresponds to the steady state, other eigenvalues with a zero real part can be obtained in two ways: either reaching the thermodynamic limit $J \rightarrow \infty$ or tuning the system into the strong-symmetry situation $\chi_{\theta}=0$. For any fixed $q, \lim _{J \rightarrow \infty} \operatorname{Re}\left[\lambda_{q, k}^{ \pm}\right]=0$, implying eigenstates with finite, purely imaginary eigenvalues. Purely imaginary eigenvalues have as a consequence the absence of stationary states and the emergence of oscillatory dynamics in the long-time limit [53], which has recently attracted attention in similar models [54,55]. This can also be observed from a mean-field analysis (see Appendix B), which in the thermal phase yields the closed orbits displayed at points (iv) and (viii) in Fig. 1(b).

\section{Dynamical symmetries}

Recently, it was shown that the absence of a stationary state and the presence of long-time oscillatory dynamics in open quantum systems can be directly implied by the existence of a dynamical symmetry operator $A$ fulfilling [53]

$$
\begin{aligned}
{[H, A] } & =\Lambda A, \\
{\left[L_{\mu}, A\right] } & =\left[L_{\mu}^{\dagger}, A\right]=0 .
\end{aligned}
$$

In that case, the matrices $\rho^{(n m)} \equiv A^{n} \rho_{\infty}\left(A^{\dagger}\right)^{m}$, with a form similar to the states that we defined in Eq. (14), are eigenvectors of the Liouvillian with purely imaginary eigenvalues

$$
\mathcal{L} \rho^{(n m)}=i(m-n) \Lambda \rho^{(n m)} .
$$

Despite the similarities, in the particular case of our model, the operator $S_{x}^{-}$does not fulfill the conditions (15) of a dynamical symmetry. However, in the $\Omega / \Gamma \gg 1$ limit, where the system is in essence purely Hamiltonian, the conditions (15) are immediately satisfied, yielding purely imaginary eigenvalues that are integer multiples of $\Omega$. We note that, in general, this will not happen for any arbitrary dissipative system in the purely Hamiltonian limit. Here the existence of a dynamical symmetry and oscillatory dynamics in the long-time limit is a consequence of having a spin Hamiltonian with equally spaced energy levels, preventing the mechanisms of eigenstate thermalization typical of closed many-body systems [80-82].

\section{DISSIPATIVE FREEZING OF THE DYNAMICS}

Having completely characterized the dissipative phases of the system and the spectral properties of the Liouvillian, we are ready to describe the effect of dissipative freezing. Several manifestations of the coexistence of multiple steady states, such as bistability and intermittency, have attracted a great deal of attention in recent years [3-6,22,26,27,30-33]. The timescale $\tau$ associated with this intermittency is related to the inverse of the ADR, which necessarily diverges at a DPT 
associated with a gapless Liouvillian. These critical phenomena, however, are typically discussed in contexts in which DPTs take place in the thermodynamic limit. Therefore, the long-time limit $\tau$ exists, at least formally, in any real finite system.

Systems with a strong symmetry differ radically from this situation, since the gap is exactly closed even for a finite system. In these cases, the dynamics is split into several unconnected symmetry sectors. In this section we describe the evolution of individual quantum trajectories of the wave function and discuss the particular situation in which the initial state is a superposition involving several of these sectors. We report the emerging phenomenon of dissipative freezing and discuss in further detail the implications of this effect in several indicators of statistics of the quantum jumps, such as the activity distribution or related quantities that appear naturally in the context of thermodynamics of quantum trajectories $[33,41,42,83-86]$.

\section{A. Freezing in individual trajectories}

Dissipative evolution of the system density matrix admits an alternative interpretation in terms of individual stochastic evolution of pure wave functions, the so-called quantum-jump or Monte Carlo wave-function approach [39]. The predictions of the master equation are recovered when one takes an ensemble average over a sufficiently high number of trajectories.

The evolution of a single trajectory can be summarized as follows. At every differential time step $d t$, for each element of the type $\left(\gamma_{i} / 2\right) \mathcal{L}_{0_{i}}[\rho]$ in the master equation, the wave function $|\psi(t)\rangle$ can randomly undergo a quantum jump with probability $p_{i}=\gamma_{i}\left\langle\psi(t)\left|\mathcal{O}_{i}\right| \psi(t)\right\rangle d t$ that transforms the system, under proper normalization, as

$$
|\psi(t+d t)\rangle \propto \mathcal{O}_{i}|\psi(t)\rangle .
$$

When no jump occurs, the wave function evolves under the action of a non-Hermitian Hamiltonian

$$
|\psi(t+d t)\rangle \propto(1-i \tilde{H} d t)|\psi(t)\rangle,
$$

where $\tilde{H} \equiv H-i \sum_{i}\left(\gamma_{i} / 2\right) \mathcal{O}_{i}^{\dagger} \mathcal{O}_{i}$. These trajectories can be physically understood as individual stochastic realizations of an experiment where quantum jumps are recorded. ${ }^{1}$ If the system is ergodic, a time average over a single trajectory also recovers the predictions of the master equation.

In the presence of a strong symmetry, the system is not ergodic and multiple degenerate steady states can exist [43]. The actual steady state of the system is then composed by a particular superposition of these states, fixed by the initial conditions $[14,34]$. However, because the evolution is not ergodic, it is not guaranteed that a single trajectory will switch among these states, which is the main assumption behind the notion of intermittency $[6,27,30-33]$. Another question that

\footnotetext{
${ }^{1}$ These unravelings are not uniquely defined, since different jump operators can be chosen that yield the same master equation (by changing the Hamiltonian accordingly). These different unravelings would correspond to different detection schemes, such as photon counting or homodyne detection, that differ on the way the system is monitored [87].
}
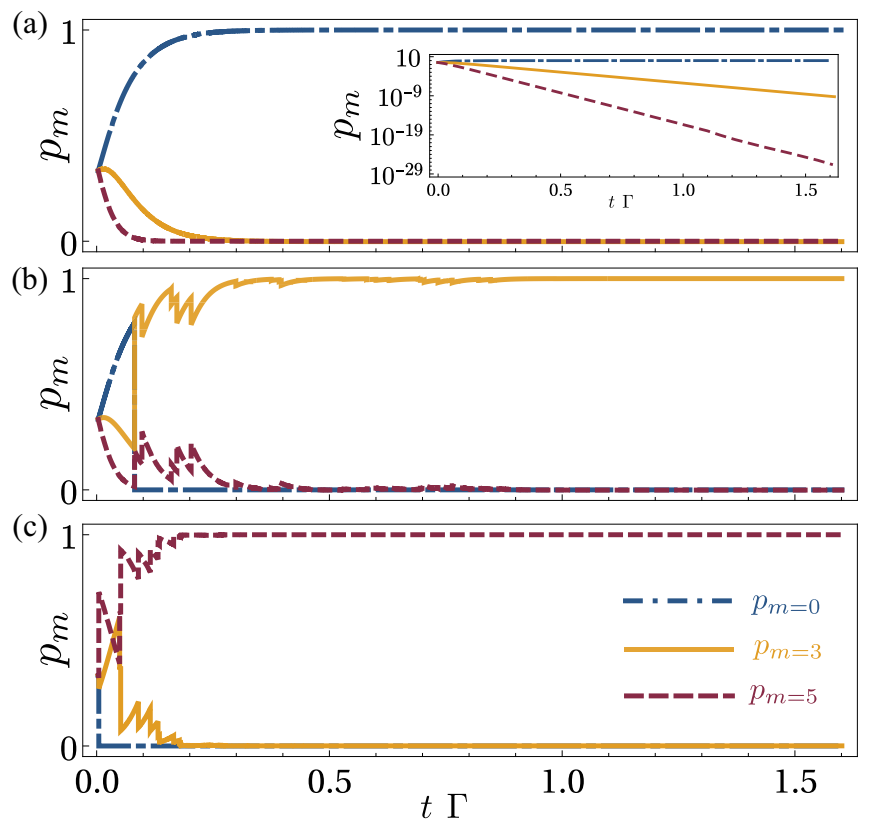

FIG. 4. (a)-(c) Three different quantum trajectories at $\theta=\pi / 4$ for the same initial state (a superposition of three eigenstates of $S_{x}$ ). The inset in (a) shows the exponential decrease of the occupation of nonselected states. The parameters are $J=5$ and $\Omega=0.8 \Gamma$.

arises is whether the conservation law associated with the strong-symmetry operator $\dot{A}=\mathcal{L}^{\dagger} A=0$ will hold at the level of individual trajectories.

In the particular case $\theta=\pi / 4$, the model of squeezed superradiance that we study here represents one of the simplest implementations of a strong symmetry, offering a privileged platform to address these questions. In order to do this, we study the quantum trajectories of states initialized in superpositions of different eigenstates of $S_{x}$. The evolution of the wave function then features what we term a dissipative freezing of the dynamics. The phenomenon is depicted in Figs. 4(a)4(c): After initializing the state in a given superposition (in this example, of the $S_{x}$ eigenstates $|0\rangle,|3\rangle$, and $|5\rangle$ ), the stochastic dissipative evolution of the wave function brings it into one of the eigenstates of $S_{x}$, with the probability of being in any of the other ones decaying exponentially with time; the evolution is effectively frozen in one eigenstate for an individual realization of the dynamics.

An eigenstate of a strong symmetry is stationary under this stochastic evolution. To prove this, we consider the general form of any wave function undergoing a stochastic dissipative evolution described by $\tilde{H}$ and the set of quantum-jump operators $\left\{L_{\mu}\right\}$. Starting from an initial state $\left|\psi\left(t_{0}\right)\right\rangle$, the wave function evolves for a time $t$ experiencing $n$ quantum jumps at times $\left(t_{1}, \ldots, t_{n}\right)<t$ with jump operators $\left(L^{(1)}, \ldots, L^{(n)}\right)$, where $L^{(i)} \in\left\{L_{\mu}\right\}$. The form of the wave function is then given by a nonunitary evolution $|\psi(t)\rangle=\frac{1}{\mathcal{N}} \tilde{U}\left(t, t_{n}, \ldots, t_{0}\right)\left|\psi\left(t_{0}\right)\right\rangle$, where $\mathcal{N}$ is a normalizing constant and $\tilde{U}\left(t, t_{n}, \ldots, t_{0}\right)$ is an evolution operator given by

$$
\tilde{U}\left(t, t_{n}, \ldots, t_{0}\right)=e^{-i \tilde{H}\left(t-t_{n}\right)} \prod_{m=1}^{n} L^{(m)} e^{-i \tilde{H}\left(t_{m}-t_{m-1}\right)},
$$


with $\prod_{m=0}^{n} \mathcal{O}_{m} \equiv \mathcal{O}_{n} \cdot \mathcal{O}_{n-1} \cdots O_{0}$. Let us consider a strong-symmetry operator $A$ so that $[A, \tilde{U}]=0$. Therefore, if $\left|\psi\left(t_{0}\right)\right\rangle$ is an eigenstate of a strong symmetry $A\left|\psi\left(t_{0}\right)\right\rangle=$ $\lambda\left|\psi\left(t_{0}\right)\right\rangle$, we obtain

$$
\begin{aligned}
A|\psi(t)\rangle & =A \tilde{U}\left(t, t_{n}, \ldots, t_{0}\right)\left|\psi\left(t_{0}\right)\right\rangle \\
& =\tilde{U}\left(t, t_{n}, \ldots, t_{0}\right) A\left|\psi\left(t_{0}\right)\right\rangle=\lambda|\psi(t)\rangle,
\end{aligned}
$$

i.e., an eigenstate of $A$ remains unchanged at the level of individual trajectories. This proof can be easily extended to the eigenstates of any power $A^{n}$. This fact may suggest that any quantum trajectory could eventually get "trapped" in one of these eigenstates, in a picture somewhat analogous to the dark-state cooling [88] or population trapping [89]. However, for this to happen, the combination of non-Hermitian Hamiltonian evolution and quantum jumps (which have opposing effects on the occupancy of each eigenstate) should bring the system into one of these eigenstates in the first place. It is $a$ priori not certain that this will occur.

Here we prove that this is indeed the case when $\dot{\rho}=$ $-i \Omega[A, \rho]+\Gamma / 2 J \mathcal{L}_{A}\{\rho\}$, i.e., dynamics with a single quantum jump $L$ and a general Hermitian strong symmetry $A \propto$ $H \propto L$. We set $t_{0}=0$ and consider an initial state $|\psi(0)\rangle=$ $\sum_{m} c_{m}(0)|m\rangle$, expanded in the basis of eigenstates of $A$, $|m\rangle$, with eigenvalue $m$. For any general quantum trajectory that evolves for a time $t$ undergoing $n$ quantum jumps, the probability for the final state to be in an eigenstate of $|m\rangle$ takes the form (see Appendix C)

$$
p(m ; t, n)=\frac{1}{\mathcal{N}}\left(e^{-|m|^{2}}|m|^{2 \alpha}\right)^{t \Gamma / J}\left|c_{m}(0)\right|^{2},
$$

with $\alpha=n J / t \Gamma$ and $\mathcal{N}$ a normalizing constant. The exponent $t \Gamma / J$ in Eq. (21) tends to enhance the maximum of the function in parentheses as time increases. Hence, after normalization, $p(m ; t, n)$ tends to zero for all $m$ except for the optimum value. Since the function $e^{-x} x^{\alpha}$ has a maximum at $x=\alpha$, only the eigenstates $|m\rangle$ from the subspace of $A^{\dagger} A$ yielding the minimum $\left.|\alpha-| m\right|^{2} \mid$ have a nonzero occupancy in the long-time limit $t \gg J / \Gamma$. Equation (21) thus encapsulates the essence of the dissipative freezing effect and is the main result of this paper: For $t \gg J / \Gamma$, any general trajectory will be trapped in an eigenspace of $A^{\dagger} A$, consequently breaking the conservation law $\dot{A}=0$ if initialized in a superposition of different eigenspaces. In the long-time limit, the total number of jumps recorded in a trajectory allows one to unambiguously determine, from those eigenspaces of $A^{\dagger} A$ having an overlap with the initial state, which one the system has been trapped in.

For the particular case that we study in this paper, $H \propto$ $L \propto A=S_{x}, m=-J, \ldots, J$. In this case, the eigenstates of $A^{\dagger} A=S_{x}^{2}$ are doubly degenerate. For $t \gg J / \Gamma$, the probability distribution for any quantum trajectory is $p(m ; t, n) \propto$ $\sum_{m}\left(\delta_{m, \tilde{m}}+\delta_{m,-\tilde{m}}\right)\left|c_{m}(0)\right|^{2}$, with $\tilde{m}$ the natural number less than or equal to $J$ closest to $\sqrt{n J / t \Gamma}$. The resulting probability distribution versus $n / t$ is plotted in Fig. 5 for an initial state composed of an equal superposition of all the eigenstates.

The phenomenon can be interpreted in terms of the quantum-measurement description that can be applied to any dissipative dynamics [90-92]. The information provided by the quantum jumps makes the eigenspaces of $A^{\dagger} A$ with a

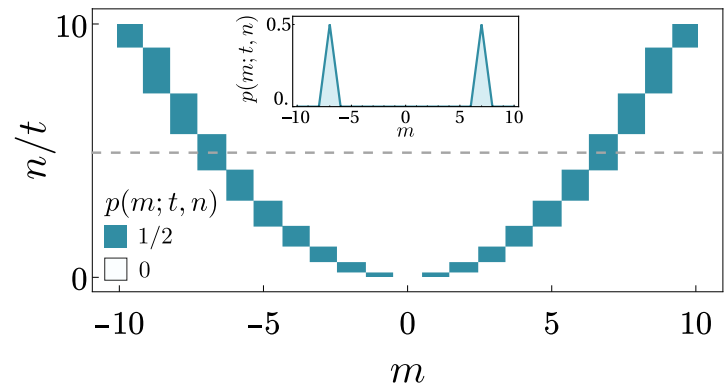

FIG. 5. (a) Probability distribution for any quantum trajectory at time $t=100 J / \Gamma$, versus the number of jumps $n$, in the model of squeezed superradiance for $\theta=\pi / 4$ and $J=10$. The initial state is an equal superposition of all the eigenstates $|m\rangle$ of $S_{x}$. The resulting wave function always freezes into an eigenstate of $S_{x}^{2}$. The inset shows the probability distribution for the value $n / t=5$ indicated by the dashed line.

particular eigenvalue increasingly likely and continuously updates the state accordingly; we note, however, that this update is different from a projective measurement of $A^{\dagger} A$ that would collapse the system into one of its eigenstates. In a standard situation, the update after each jump will not tend to freeze the state, due to the non-Hermitian evolution between jumps, which changes the occupancy of these eigenstates and dissolves the effect of the jump. In our case, this is prevented by the strong symmetry, and the effect of the jumps accumulates, giving rise to the phenomenon of dissipative freezing.

\section{B. Activity distribution}

Now that we have presented the dissipative freezing effect, it is instructive to analyze it in terms of one of the main observables of interest when discussing multistability: the activity $[33,83]$. The activity is defined as the mean number of quantum jumps undergone by the system per unit time; this can be defined through the probability distribution $p_{T}(K)$ of counting $K$ jumps over a time $T$. Following our previous discussion, we assume the existence of a strong symmetry $A$ with eigenstates $|m\rangle$ and only one quantum-jump operator $L=\sqrt{\Gamma / J} A$. We consider an initial state with the form

$$
\rho(0)=\sum_{m} c_{m}|m\rangle\langle m|
$$

This initial state is a steady state of the system, meaning that its preparation can always be conceived as the long-time limit of another initial state. Other choices of $\rho(0)$ may involve transient effects that will be irrelevant in the limit $T \rightarrow \infty$. We can then prove (see Appendix D) that the photon-counting distribution takes the form

$$
p_{T}(K)=\sum_{m} \frac{1}{K !}\left(\frac{T \Gamma m^{2}}{J}\right)^{K} e^{-\Gamma m^{2} T / J} c_{m},
$$

which is dependent on the initial state. This equation presents the multimodal structure depicted in Fig. 6(a), where we plot it for the particular case of our model, where $A=S_{x}$. The physical interpretation is simple: With every eigenstate $|m\rangle$ 

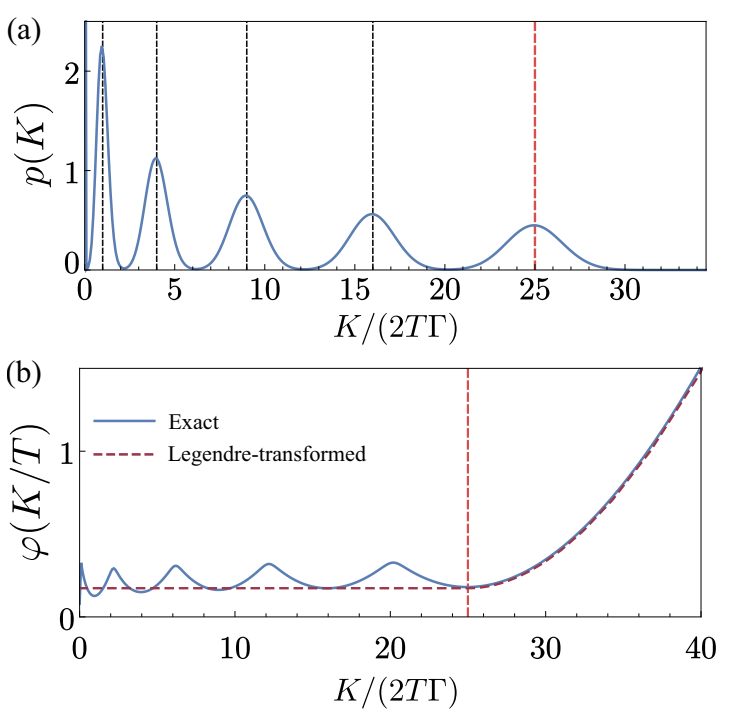

FIG. 6. (a) Probability of having $K$ quantum jumps in a time $T=$ $3 \times 10^{3} / \Gamma$ in the case of our model, where $A=S_{x}$, for $N=20, \Omega=$ $0.8 \Gamma$, and $\theta=\pi / 4$ (strong-symmetry point). (b) Rate function $\varphi(k)$, obtained directly from the logarithm of Eq. (23) (blue solid line) and by a Legendre transformation (red dashed line). The Legendre transformed $\varphi(k)$ is given by Eq. (32) plus an additive constant to match the normalization of $p(K)$ for a finite $T$.

of $A$, there is an associated steady state

$$
\rho_{0}^{(m)}=|m\rangle\langle m|,
$$

with a corresponding quantum-jump rate of $\operatorname{Tr}\left[L^{\dagger} L \rho\right]=$ $m^{2} \Gamma / J$. The set of $\rho_{0}^{(m)}$ forms a basis, meaning that any combination of these steady states is a also steady state. The asymptotic state

$$
\rho_{\mathrm{ss}}=\lim _{t \rightarrow \infty} e^{\mathcal{L} t} \rho(0)=\sum_{m} \operatorname{Tr}\left[\rho_{0}^{(m)}, \rho(0)\right] \rho_{0}^{(m)},
$$

is therefore strongly dependent on the initial state and given by its overlap with each of the $\rho_{0}^{(m)}$. Those $\rho_{0}^{(m)}$ having a finite overlap with $\rho(0)$ will manifest as a distinct peak in the counting distribution $p_{T}(K)$, centered at the value $K_{m}=$ $T m^{2} \Gamma / J$.

Multimodality (as a signature of multistability) has been recently associated with dynamical phase transitions [33] that feature the coexistence of two phases in time, with a stochastic switching between these phases that has been observed experimentally on multiple occasions [6,27,30-32]. While we obtain a clear multimodal structure for the activity distribution, our results on the dissipative freezing do not match this notion of intermittency. Let us therefore put our results in the context of the theory used in Ref. [33]: the thermodynamics of quantum trajectories.

\section{Thermodynamics of quantum trajectories: Dynamical phase transition \\ 1. Brief introduction to the thermodynamics of quantum trajectories}

Recently, several works [33,41,42,83-86] have approached the questions of multimodality and intermittency from the perspective of the thermodynamics of quantum trajectories. This approach regards the set of quantum trajectories in which the dynamics can be unraveled as a statistical ensemble that can be analyzed using the tools of statistical mechanics. In the following, we briefly outline this theory (a comprehensive description can be found in Refs. [33,83]) and discuss its implications in systems, such as the one we report here, where dissipative freezing of the dynamics occurs.

Let us consider a system governed by the master equation $\dot{\rho}=\mathcal{L} \rho=-i[H, \rho]+L \rho L^{\dagger}-\frac{1}{2}\left\{L^{\dagger} L, \rho\right\}$. The evolution of $\rho$ can be unraveled as a set of quantum trajectories [38-40] by which a conditional density matrix $\rho_{K}(t)$ can be built from the ensemble average of all the trajectories of duration $t$ having $K$ quantum jumps. The activity distribution is then given by $p_{K}(t)=\operatorname{Tr} \rho_{K}(t)$. We can define a generating function $Z=$ $\left\langle e^{s K}\right\rangle$,

$$
Z=\sum_{K=0}^{\infty} e^{s K} p_{K}(t)=\operatorname{Tr} \sum_{K=0}^{\infty} e^{s K} \rho_{K}(t)=\operatorname{Tr} \rho_{s}(t),
$$

where $\rho_{s}(t) \equiv \sum_{K=0}^{\infty} e^{s K} \rho_{K}(t)$ is a Laplace transformed density matrix that evolves according to a tilted master equation

$$
\mathcal{W}_{s} \rho_{s}=\dot{\rho}_{s}=-i\left[H, \rho_{s}\right]+e^{s} L \rho L^{\dagger}-\frac{1}{2}\left\{L^{\dagger} L, \rho\right\}
$$

and the counting field $s$ is a variable conjugate to $K$. For $s=0$, Eq. (27) corresponds to the normal master equation $\mathcal{W}_{s}=\mathcal{L}$. For $s \neq 0$, Eq. (27) is not a physical trace-preserving master equation and describes a class of dynamics in which the quantum jumps are biased by the factor $e^{s}$. Despite $\mathcal{W}_{s}$ being unphysical, its spectral properties contain valuable information about the fluctuations of the ensemble of trajectories. In particular, the partition function acquires, in the longtime limit, a large deviation form $Z \asymp e^{t \lambda(s)}$, with $\lambda(s)$ the eigenvalue of $\mathcal{W}_{s}$ with the largest real part. This allows us to write the activity or mean emission rate as

$$
\langle k\rangle=\langle K\rangle / t=\left.\frac{1}{t} \frac{\partial Z}{\partial s}\right|_{s=0}=\left.\frac{\partial \lambda(s)}{\partial_{s}}\right|_{s=0} .
$$

This suggests the definition of an $s$-dependent emission rate $\langle k\rangle_{s} \equiv \partial \lambda / \partial s(s)$. Equivalently, fluctuations in the activity can be described by Mandel's $Q$ parameter $Q=\left(\left\langle K^{2}\right\rangle-\right.$ $\left.\langle K\rangle^{2}\right) /\langle K\rangle-1$, given by

$$
Q=\left.\frac{\partial^{2} \lambda / \partial s^{2}}{\partial \lambda / \partial s}\right|_{s=0}
$$

To sum up, the behavior of $\lambda(s)$ around the vicinity of $s=$ 0 characterizes the fluctuations of the ensemble of quantum trajectories.

The connection to thermodynamics put forward in Ref. [83] can be made by assuming that, in the long-time limit, $p_{K}(t)$ also acquires a large-deviation form

$$
p_{K}(t) \asymp e^{-t \varphi(K / t)} .
$$

If $p_{K}(t)$ describes the probability distribution of an statistical ensemble, then the rate function $\varphi(K / t)=-\ln p_{K}(t) / t$ plays the role of an entropy density [93]. By plugging Eq. (30) into Eq. (26), we obtain directly that $\varphi(k=K / t)$ and $\lambda(s)$ are 
related by a Legendre transformation

$$
\lambda(s)=\max _{k}[k s-\varphi(k)],
$$

meaning that $\lambda(s)$ has the properties of a free energy. The inverse transformation

$$
\varphi(k)=\max _{s}[k s-\lambda(s)]
$$

is a useful relation that allows us to obtain $\varphi(k)$ from the knowledge of $\lambda(s)$, which can in turn be computed from the eigenvalues of $\mathcal{W}_{s}$. However, this relation follows from the Gärtner-Ellis theorem [93], which requires $\lambda(s)$ to be differentiable for all $s \in \mathbb{R}$ or, equivalently, $\varphi(k)$ to be concave for all $k \in \mathbb{R}$. These are precisely the conditions that are violated when a phase transition occurs.

\section{Multistability and breaking of the intermittency: Connection to known models}

In Ref. [33], the coexistence of dynamical phases was linked to a discontinuity in the $s$-dependent order parameter $\langle k\rangle_{s}$ at the physical point $s=0$, i.e., a first-order phase transition with respect to the counting field. Based on this temporal coexistence between phases, such a first-order phase transition was then referred to as a dynamical phase transition. As we show in Fig. 7(a), where we plot a numerical calculation of $\langle k\rangle_{s}$ versus $\theta$, the closing of the gap at the strong-symmetry point gives rise to such a discontinuity; the limit $s \rightarrow 0^{+}$features a bright phase characterized by a high activity, whereas for $s \rightarrow 0^{-}$we find a dark phase with virtually no quantum jumps. The discontinuity turns into a continuous crossover as we depart from the point $\theta=\pi / 4$, consistent with a firstorder phase transition smoothed by finite-size effects. Such a crossover is responsible for the phenomenon of intermittency typically observed in finite many-body systems undergoing a DPT [33]. When the crossover turns into a real discontinuity, here due to the appearance of a strong symmetry, intermittency is replaced by the phenomenon of dissipative freezing.

We elaborate on this argument by proving first that a strong symmetry implies that $\langle k\rangle_{s}$ is discontinuous (a similar analysis was performed in Ref. [35]). Following our previous discussion, we focus on the case where a strong symmetry $A$ is present and $L=\sqrt{\Gamma / J} A$. We can immediately see that the steady states $\rho_{0}^{(m)}$ in Eq. (24) are also eigenstates of $\mathcal{W}_{s}$, with eigenvalues

$$
\lambda^{(m)}(s)=\frac{\Gamma}{J} m^{2}\left(e^{s}-1\right) .
$$

Since these are the largest eigenvalues for $s=0$, they must also be in the vicinity of that point. Therefore, we can write $\lambda(s)$ around $s=0$ as

$$
\lambda(s)= \begin{cases}(\Gamma / J) m_{\min }^{2}\left(e^{s}-1\right), & s<0 \\ (\Gamma / J) m_{\max }^{2}\left(e^{s}-1\right), & s>0,\end{cases}
$$

with $m_{\min (\max )}$ the minimum (maximum) eigenvalues of $A$. If $m_{\min } \neq m_{\max }$, it is clear that $\lambda(s)$ shows a singular behavior at $s=0$, having a discontinuous derivative. Contrary to the situations typically considered, the discontinuity does not become a crossover when the system has a finite size, since its origin is the exact closing of the Liouvillian gap due to
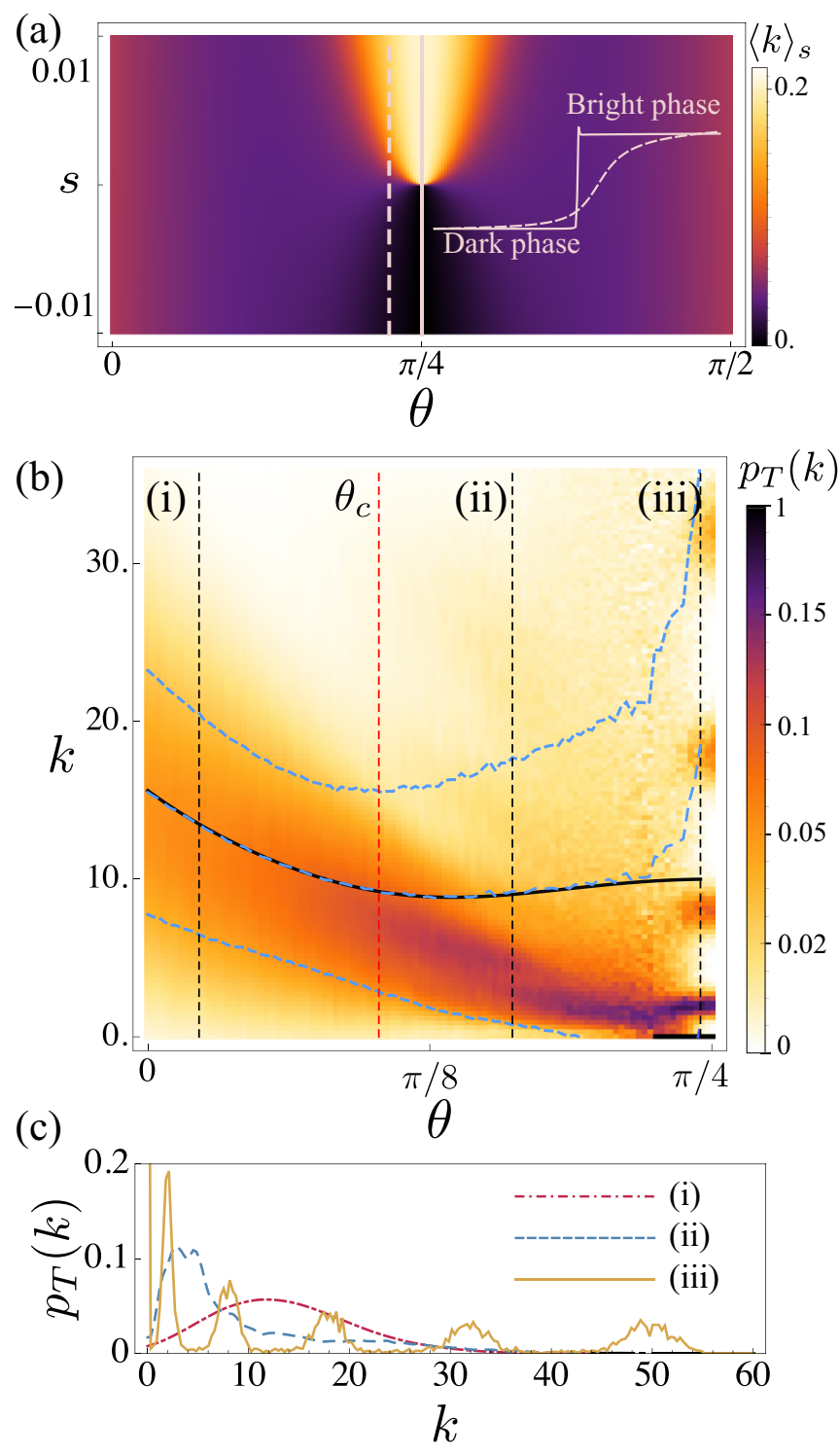

FIG. 7. (a) Plot of $\langle k\rangle_{s}$ versus $\theta$, featuring the coexistence between a bright and a dark phase in the vicinity of $\theta=\pi / 4$. (b) Probability distribution $p_{T}(k)$ of the activity versus the squeezing angle $\theta$ for $\Omega=0.8 \Gamma$. The distribution for each $\theta$ has been computed from 400 Monte Carlo trajectories. The time $T$ has been taken in each case as half the relaxation time $\tau=1 /\left|\lambda_{1}\right|$. Points (i)-(iii) indicate the three values of $\theta$ shown in (c). The blue line (black dashed line) corresponds to $\langle k\rangle$ calculated from the master equation (Monte Carlo trajectories); blue dashed lines correspond to the variance $\Delta k^{2}$ computed from the Monte Carlo trajectories.

the strong symmetry (see Fig. 3). If we were to try to find $\varphi(k)$ by blindly applying Eq. (32) with a generic expression for $\lambda(s)=(\Gamma / J) m^{2}\left(e^{s}-1\right)$, we would find that the value of $s$ that maximizes $k s-\lambda(s)$ is given by

$$
s= \begin{cases}\ln \left(J k / \Gamma m_{\min }^{2}\right), & k<\frac{\Gamma}{J} m_{\min }^{2} \\ \ln \left(J k / \Gamma m_{\max }^{2}\right), & k>\frac{\Gamma}{J} m_{\max }^{2} \\ 0, & \frac{\Gamma}{J} m_{\min }^{2}<k<\frac{\Gamma}{J} m_{\max }^{2} .\end{cases}
$$

This yields the rate function shown by the red dashed line in Fig. 6(b): The nonconcave regions of $\varphi(K / t)$ associated 
with multimodality translate into a nonphysical flat plateau when one tries to use the inverse Legendre transformation in Eq. (32). This result connects back to standard thermodynamics, where phase transitions are associated with nonconcavities in the underlying fundamental equations for the thermodynamic potentials. A multimodal distribution $p_{T}(k)$ as we obtained in Eq. (23) will always yield a discontinuous $\lambda(s)$ and will therefore be linked to a first-order phase transition.

To summarize, we have discussed the notions of dissipative freezing (21), multimodal activity distributions (23), and first-order phase transitions at the trajectory level (35). We conclude that these phenomena are linked, since all of them emerge from the existence of a strong symmetry that yields a perfect closing of the Liouvillian gap for any system size. Intermittency is therefore a consequence of the finite system size; it implies a smoothing of the phase transition that allows us to make use of Eq. (32), but that gives in turn a unimodal probability distribution; i.e., in the long-time limit, intermittency destroys multimodality [24]. Dissipative freezing can therefore be alternatively described as the survival of multimodality in the long-time limit. In quantum metrology, this has strong implications for the scaling in time of the Fisher information [24].

These ideas are further supported by numerical calculations in Figs. 7(b) and 7(c), where we show $p_{T}(k)$ computed from sets of quantum trajectories, for time windows approximately twice the inverse Liouvillian gap $T \approx\left(2 \operatorname{Re}\left\{\lambda_{2}\right\}\right)^{-1}$. The value of $\Omega=0.8 \Gamma$ is such that we can observe the transition from the ferromagnetic to the thermal phase at $\theta_{c} \approx 0.4$. When this transition is crossed, fluctuations start increasing with $\theta$ [see the blue dashed lines in Fig. 7(b)] and the unimodal distribution is strongly distorted. This characteristic of the thermal phase is the consequence of the increased asymmetry on $\langle k\rangle_{s}$ at $s=0$ [see Fig. 7(a)], which is associated with the closing of the Liouvillian gap. As we get close to $\pi=\pi / 4$, where $\operatorname{Re}\left\{\lambda_{2}\right\}=0$, it becomes impossible to simulate times of the order of $\operatorname{Re}\left\{\lambda_{2}\right\}^{-1}$. In the plot, this is identified by the emergence of several peaks in $p_{T}(k)$ : The crossover in $\langle k\rangle_{s}$ gives rise to a multipeaked structure that would merge into a single peak were $T$ long enough. Since this multimodality does not correspond to the long-time limit, the large-deviation approach is unable to describe it; this is the situation in which intermittency occurs. On the other hand, the strong-symmetry point features a multimodal $p_{T}(k)$ for any $T$; that survival of the multimodal structure is the signature of dissipative freezing of the dynamics.

The survival of multimodality is of great importance in the context of enhanced quantum metrology, where it has been proven that there is a Heisenberg scaling of the quantum Fisher information for times shorter than the correlation time $\tau$ [24]. Since systems with a strong symmetry will feature an asymptotic quadratic scaling of the quantum Fisher information for all times, our results may be of relevance in the design of sensing protocols aimed to exploit this feature in continuous Bayesian parameter estimation from photon counting $[92,94,95]$. Beyond the model considered here, our results have significant implications for the dynamical characterization of DPTs in more complex systems where the existence of a strong symmetry can provide a way to tune

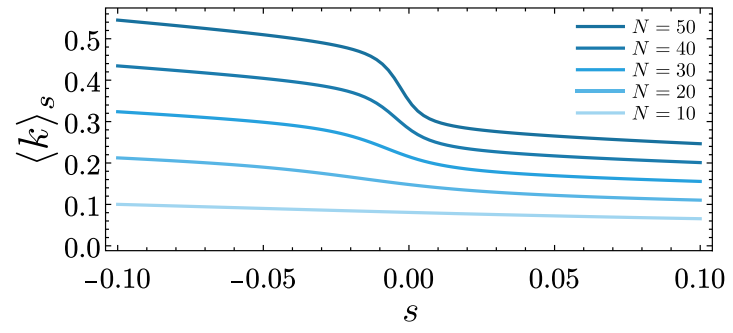

FIG. 8. Emergence of a crossover in the $s$-dependent activity parameter with increasing system size at $\theta=0$ for $\Omega=4 \Gamma$.

the Liouvillian gap to zero without the need of reaching a thermodynamic limit.

Finally, we note that the closing of the Liouvillian gap in the thermodynamic limit of the thermal phase [cf. Eq. (13)] also yields a crossover in $\langle k\rangle_{s}$ (see Fig. 8). Since this closing is of a different nature (associated with eigenvalues with imaginary part), it offers the interesting prospect of studying multistability and intermittency between phases displaying coherent oscillatory dynamics in the long-time limit. This could be done, for instance, by analyzing the time correlations between the spectral features of the different phases, as we discuss in the following section.

\section{SIGNATURES OF CRITICAL DYNAMICS IN THE EMITTED LIGHT}

In this section we discuss the possibility of probing some of the essential features of the low-energy spectrum of the Liouvillian by analyzing the light emitted by the system. Many of the essential features of critical dissipative dynamics are encoded in the spectral properties of the Liouvillian. Stationary observables of the form $\langle\mathcal{O}\rangle=\operatorname{Tr}\left[\mathcal{O} \rho_{0}\right]$ contain a limited amount of information about these properties, since they depend only on the lowest eigenvalue of $\mathcal{L}$. However, observables involving two-time correlators of the form $\langle\mathcal{O}(t) \mathcal{O}(t+\tau)\rangle$ require knowledge not only of $\rho_{0}$, but also of the Liouvillian $\mathcal{L}$. Consequently, they carry information about the dynamics of the system that is not present $\rho_{0}$ and can provide valuable data about $\mathcal{L}$, such as its spectral properties, in an experimentally accessible way. To illustrate this point, we focus here on the case of the spectrum of emission, providing a closed-form expression in terms of the Liouvillian eigenvalues and right and left eigenstates.

We define the (unnormalized) spectrum of emission as

$$
S(\omega)=\lim _{t \rightarrow \infty} \frac{1}{\pi} \operatorname{Re} \int_{0}^{\infty} d \tau e^{i \omega \tau}\left\langle a^{\dagger}(t) a(t+\tau)\right\rangle,
$$

where, generally, $a$ is some system operator linked to the bath output operator by input-output relations (in our case, $a=D_{\theta}$ ). By applying the quantum regression theorem [96], we obtain

$$
S(\omega)=\lim _{t \rightarrow \infty} \frac{1}{\pi} \operatorname{Re} \int_{0}^{\infty} d \tau e^{i \omega \tau} \operatorname{Tr}\left\{a e^{\mathcal{L} \tau}\left[\rho(t) a^{\dagger}\right]\right\} .
$$

Note that, typically, the limit $t \rightarrow \infty$ will imply that $\rho(t)$ is simply $\rho_{0}$, the steady state of the system. In most systems this steady state is unique, but here we want to take into account the possibility of multiple steady states (i.e., multiple 
(a)

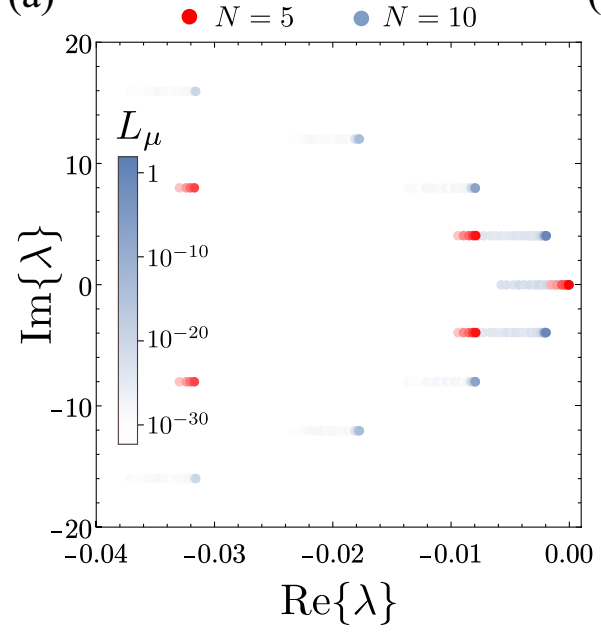

(b)

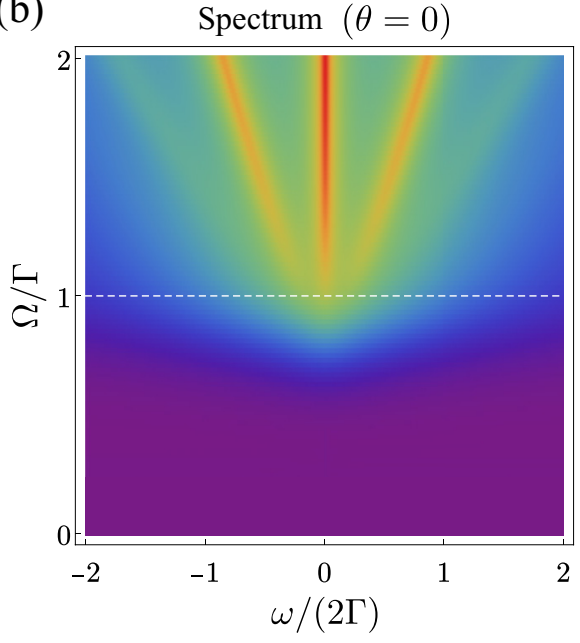

(c)

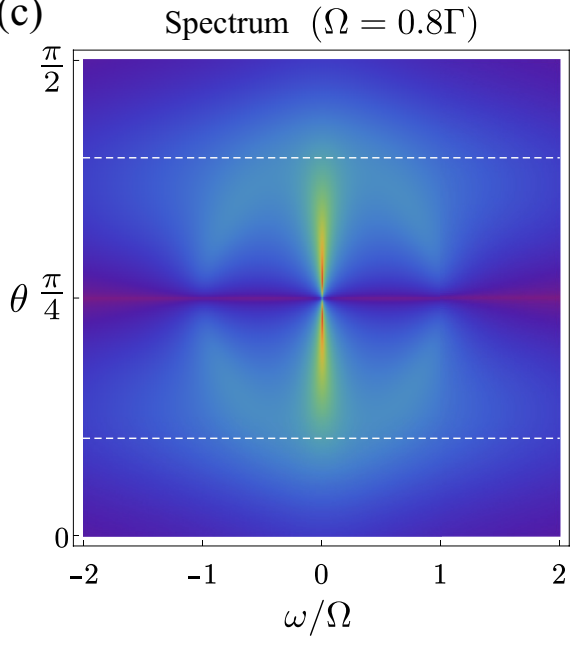

FIG. 9. (a) Liouvillian eigenvalues $\lambda_{\mu}$ weighted by $L_{\mu}$. This illustrates the set of eigenvalues that is experimentally accessible by the measurement of the spectrum of emission. (b) Spectrum of emission versus $\Omega$, for $\theta=0$. (c) Spectrum versus $\theta$, for $\Omega=0.8 \Gamma$. At the strong-symmetry point, the gap closes exactly, and the spectrum features an extreme line narrowing. White dashed lines indicate where a phase transition occurs; the signature of the phase transition is the emergence of sideband peaks.

eigenstates of $\mathcal{L}$ with eigenvalues with zero real part), meaning that $\rho(t)$ can be any superposition of these steady states, defined by the initial state. Therefore, we take the limit $t \rightarrow$ $\infty$ and replace $\rho(t)$ by an arbitrary superposition of steady states $\rho_{\mathrm{ss}}$ determined by the initial state. We can perform a spectral decomposition of the Liouvillian to write, for any $\rho$,

$$
e^{\mathcal{L} t} \rho=\sum_{\mu} e^{\lambda_{\mu} t} \operatorname{Tr}\left[\rho_{L, \mu} \rho\right] \rho_{R, \mu},
$$

where $\rho_{L(R), \mu}$ is the left (right) eigenstate of $\mathcal{L}$ with eigenvalue $\lambda_{\mu}$. This allows us to write

$$
S(\omega)=\frac{1}{\pi} \operatorname{Re} \int_{0}^{\infty} d \tau \sum_{\mu} e^{\left(i \omega+\lambda_{\mu}\right) \tau} \operatorname{Tr}\left[a \rho_{R, \mu}\right] \operatorname{Tr}\left[a^{\dagger} \rho_{L, \mu} \rho_{\mathrm{ss}}\right] .
$$

By defining

$$
\begin{aligned}
\omega_{\mu} & \equiv \operatorname{Im} \lambda_{\mu}, \\
\gamma_{\mu} / 2 & \equiv-\operatorname{Re} \lambda_{\mu}, \\
L_{\mu} & \equiv \operatorname{Re} \operatorname{Tr}\left[a \rho_{\mathrm{R}, \mu}\right] \operatorname{Tr}\left[a^{\dagger} \rho_{\mathrm{L}, \mu} \rho_{\mathrm{ss}}\right], \\
K_{\mu} & \equiv \operatorname{Im} \operatorname{Tr}\left[a \rho_{\mathrm{R}, \mu}\right] \operatorname{Tr}\left[a^{\dagger} \rho_{\mathrm{L}, \mu} \rho_{\mathrm{ss}}\right],
\end{aligned}
$$

we can formally integrate Eq. (39) to obtain

$$
\begin{aligned}
S(\omega)= & \frac{1}{\pi} \sum_{\mu, \operatorname{Re} \lambda_{\mu} \neq 0} \frac{\left(\gamma_{\mu} / 2\right) L_{\mu}-\left(\omega+\omega_{\mu}\right) K_{\mu}}{\left(\gamma_{\mu} / 2\right)^{2}+\left(\omega+\omega_{\mu}\right)^{2}} \\
& +\sum_{\mu, \operatorname{Re} \lambda_{\mu}=0}\left[L_{\mu} \delta\left(\omega+\omega_{\mu}\right)+\frac{K_{\mu}}{\pi} \mathrm{P} \cdot \mathrm{V} \cdot\left(\frac{1}{\omega+\omega_{\mu}}\right)\right] .
\end{aligned}
$$

Note that terms with $\operatorname{Re} \lambda_{\mu}=0$ give rise to a series of $\delta$ peaks in the spectrum, positioned at frequencies that are given by the imaginary part of the eigenvalues with a zero real part. The last term means that the principal value integral of $1 /(\omega+$ $\left.\omega_{\mu}\right)$ should be computed when integrating that distribution.
That term never appears in the case of a unique steady state $(\mu=0)$, since in that case $\rho_{L, 0}=\mathbb{1}$ and $K_{0}=\operatorname{Im}\left|\langle a\rangle_{\mathrm{ss}}\right|^{2}=0$. All the terms proportional to $K_{\mu}$ in Eq. (41) are dispersive line shapes that break the symmetry of the corresponding Lorentzians (proportional to $L_{\mu}$ ). Although they may appear unphysical (since they can yield negative values), they give a physical result once the sum is performed.

Equation (41) tells us that the spectrum of emission can be used to probe the Liouvillian spectrum and also infer, indirectly, information about the right and left eigenvectors. Similar formal integrations of Eq. (37) have been presented before [97-99]; ours differs from those in that they make explicit use of the left and right eigenvectors of $\mathcal{L}$. In particular, we see that the existence of eigenvalues with a zero real part and a finite imaginary part translates into the presence of measurable $\delta$ peaks in the spectrum. These turn into peaks with a finite width when the linewidth of the detectors and/or other unavoidable losses to different channels are included in the description. Figure 9 illustrates the information about the Liouvillian eigenvalues provided by the spectrum in the model discussed in this work. Figure 9(a) shows the distribution of eigenvalues for $N=(5,10)$, weighted by their value of $L_{\mu}$. This way, features like the emergence of imaginary eigenvalues with a vanishing real part in the thermodynamic limit can be directly measured in the laboratory. We show this in Fig. 9(b), where the ferromagnetic-thermal DPT is shown to be accompanied by the emergence of sideband peaks in the fluorescence spectrum; this is the well-known generalization of the Mollow triplet to the case of collective resonance fluorescence [100]. The result that the Liouvillian gap closes in this phase as $1 / N$ can be confirmed experimentally: As shown in Fig. 10, it can be measured directly as a decrease in the linewidth of the spectral peaks. Finally, Fig. 9(c) shows the emergence of sideband peaks when $\theta$ is varied so as to enter the thermal phase and the observation of extreme line narrowing as the gap is closed exactly at the strong-symmetry point. 


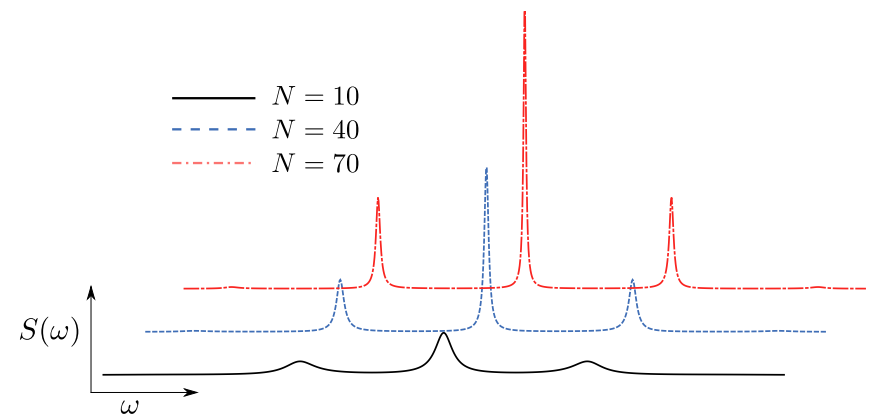

FIG. 10. Spectrum of emission $S(\omega)$ in the thermal phase for different values of $N$. The fact that the real part of the highest eigenvalues goes to zero as $1 / N$ can be measured as a narrowing of the spectral peaks. The parameters are $\theta=0$ and $\Omega=2 \Gamma$.

These results open the intriguing possibility of exploring the notions of ergodicity, intermittency, and dissipative freezing in systems with Liouvillian eigenvalues with a vanishing real part and a finite imaginary part by studying temporal correlations between different spectral windows [98,101-107]. This is a topic of study left for future work.

\section{CONCLUSION}

We have studied the model of squeezed superradiance and analyzed the different types of nonergodic dynamics emerging in dissipative phases with a gapless Liouvillian. In order to identify the relevant regimes of nonergodic dynamics, we have completely characterized the phase diagram of the system, its metrological properties, and its Liouvillian spectrum. We have shown the existence of nonstationary dynamics linked to Liouvillian eigenvalues with a finite imaginary part and a vanishing real part in the thermodynamic limit and we have reported the phenomenon of dissipative freezing that appears when the Liouvillian has a strong symmetry. We have connected the phenomenon of dissipative freezing with the theory of thermodynamics of quantum trajectories, showing that it is linked to a real discontinuity in the associated first-order phase transition with respect to the counting field. Intermittency is, on the other hand, linked to the smoothing of such first-order phase transition into a crossover due to finite-size effects. Notably, the model studied here allows us to explore all this phenomenology with a finite-size system that can be treated numerically. Our work sheds light on the critical behavior of open systems with finite system size and provides useful insights for the design and characterization of sensors based on the critical behavior of driven-dissipative quantum systems $[24,25,92,94,95]$.

\section{ACKNOWLEDGMENTS}

C.S.M. gratefully acknowledges F. Minganti for fruitful and insightful discussions. B.B. and C.S.M. are grateful to Juan P. Garrahan for very useful comments and insightful discussions. C.S.M. was funded by the Marie SkłodowskaCurie Fellowship QUSON (Project No. 752180). B.B., J.T., and D.J. acknowledge support from EPSRC Grants No. EP/P009565/1 and No. EP/K038311/1 and from the European Research Council under the European Union's Sev- enth Framework Programme (Program No. FP7/2007-2013), ERC Grant Agreement No. 319286 Q-MAC. A.G.-T. and D.P. acknowledge support from CSIC Research Platform on Quantum Technologies PTI-001 and from Spanish Project No. PGC2018-094792-B-100 (MCIU/AEI/FEDER, EU).

\section{APPENDIX A: MEAN-FIELD EQUATIONS}

The study of mean-field equations provides insight into the system dynamics and the different dissipative phases in the thermodynamic limit $J \rightarrow \infty$. In that case, writing the commutator between the normalized angular momentum operators $s_{i} \equiv S_{i} / J, i \in\{x, y, z\}$, yields a value $\left[s_{i}, s_{j}\right]=i \epsilon^{i j k} s_{k} / J$ (with $\epsilon^{i j k}$ the Levi-Cività symbol) that tends to zero. One thus obtains the set of equations

$$
\begin{aligned}
& \dot{s}_{x}=\left(\Gamma_{-}-\Gamma_{+}\right) s_{x} s_{z}, \\
& \dot{s}_{y}=-\Omega s_{z}+\left(\Gamma_{-}-\Gamma_{+}\right) s_{y} s_{z}, \\
& \dot{s}_{z}=\Omega s_{y}-\left(\Gamma_{-}-\Gamma_{+}\right)\left(s_{x}^{2}+s_{y}^{2}\right),
\end{aligned}
$$

where $\Gamma_{ \pm}$are given by Eqs. (4a) and (4b). At the level of description of the mean-field equations, the role of the squeezing angle $\theta$ is therefore to renormalize the decay rate $\Gamma$ by the factor $\left(\cos ^{2} \theta-\sin ^{2} \theta\right)$, since $\Gamma_{-}-\Gamma_{+}=\Gamma\left(\cos ^{2} \theta-\sin ^{2} \theta\right)$. Given that these equations conserve the total norm $\mathcal{N}=s_{x}^{2}+$ $s_{y}^{2}+s_{z}^{2}$, we can write them as a reduced set of dynamical equations in terms of the polar angles $\{\Theta \in[0, \pi], \Phi \in[-\pi, \pi]\}$, related to the Cartesian coordinates as

$$
\begin{aligned}
& s_{x}=\sin \Theta \cos \Phi, \\
& s_{y}=\sin \Theta \sin \Phi, \\
& s_{z}=-\cos \Theta .
\end{aligned}
$$

Note that our definition differs from the standard one by the sign of Eq. (A2c), which means that the angle $\Theta$ is defined with respect to the $-z$ axis in order to make the lowest eigenstate of $S_{z}$ correspond to $\Theta=0$. The dynamical equations for the spherical angles are

$$
\begin{aligned}
& \dot{\Theta}=\Omega \sin \Phi-\left(\Gamma_{-}-\Gamma_{+}\right) \sin \Theta, \\
& \dot{\Phi}=\Omega \cos \Phi \cot \Theta .
\end{aligned}
$$

These equations define a vector field of derivatives on the Bloch sphere; these field lines are sketched in Fig. 1(b) for different values of $(\Omega / \Gamma, \theta)$, together with the spin Wigner function $[70,71]$ of the exact steady state in a finite system $(N=50)$. A mean-field approach does not necessarily offer a faithful description of the dynamics [22]; in our model, it assumes a classical pointlike state on the Bloch sphere, therefore failing to describe spin fluctuations. Despite this, it is interesting to notice that, in a finite system, the shape of the fluctuations in the Bloch sphere actually bears some similarities to the vector field of derivatives predicted by the mean field [108]. This is observed in Fig. 1, where it can be clearly seen that the asymmetry in the density of field lines at both sides of the steady state (moving along the meridian) is replicated as an asymmetry in the corresponding Wigner function.

We move now to analyzing the steady solutions of these dynamical equations. Regarding the angle $\Phi$, Eq. (A3b) 
always has a stationary solution at $\Phi= \pm \pi / 2$. It is instructive to consider the dynamics of $\Theta$ for $\Phi=\pi / 2$, which reduces to

$$
\dot{\Theta}=\Omega-\left(\Gamma_{-}-\Gamma_{+}\right) \sin \Theta .
$$

One can picture this as a dynamical equation for a pendulum, driven by the first term and damped by the second. The steady-state solution $\Theta_{0}$ is determined by setting (A4) to zero, which, from Eq. (A2), yields the magnetization $M \equiv s_{z}(t \rightarrow$ $\infty)$ given by Eq. (7). In general, the stationary solutions of Eqs. (A1a)-(A1c) read

$$
\begin{aligned}
& \left\langle s_{z}\right\rangle=M, \\
& \left\langle s_{x}\right\rangle=0, \\
& \left\langle s_{y}\right\rangle=\sqrt{1-M^{2}} .
\end{aligned}
$$

There are two situations in which these solutions do not hold.

(i) At the point $\Gamma_{-}-\Gamma_{+}=0$, where $S_{x}$ becomes a strong symmetry, Eq. (A4) does not have a stationary solution except for the trivial case $\Omega=0$. In particular, looking back at Eqs. (A1a)-(A1c), we see that at this point the evolution corresponds to a circular motion on a plane of constant $s_{x}$, with $s_{y}=\left(1-s_{x}^{2}\right) \cos (\Omega t)$ and $s_{z}=\left(1-s_{x}^{2}\right) \sin (\Omega t)$.

(ii) At the critical value

$$
\Omega_{c}=\Gamma_{-}-\Gamma_{+}=\Gamma\left(\cos ^{2} \theta-\sin ^{2} \theta\right),
$$

we have $M=0$, which means that the energy supplied by $\Omega$ is enough to reach the equator of the Bloch sphere, where the drag is maximum.

Therefore, for values $\Omega>\Omega_{c}$, the pendulum is able to go beyond the equator, with a driving that now is large enough for it to engage in a perpetual oscillation across the Bloch sphere. This is reflected in the fact that Eq. (A4) has no stationary solution and in the unphysical imaginary value of $M$ predicted by Eq. (7) for $\Omega>\Omega_{c}$. The emergence of initialstate-dependent closed trajectories at $\Omega>\Omega_{c}$ is represented at points (iv) and (viii) in Fig. 1(b). This transition to a phase with time-periodic steady states corresponds, in the case $\theta=0$, to the well-studied second-order DPT of collective resonance fluorescence $[52,68,69]$, it is related to the existence of steady states with imaginary eigenvalues [53], and it was the subject of recent work $[54,55]$ where similar models were used to describe dissipative time crystals.

In general, we observe that the role of the squeezed decay parametrized by $\theta$ is to lower the value of critical driving towards $\Omega_{c} \rightarrow 0$ as $\theta \rightarrow \pi / 4$ (and $\Gamma_{-} \rightarrow \Gamma_{+}$). Note that such an apparent nonergodic dynamics does not survive in the full quantum solution for a finite system, which does reach stationarity on a time that diverges, however, with the system size [as predicted by the eigenvalue equation (13)]. The stationary oscillations predicted by the mean-field equations are therefore the thermodynamic limit of a transient phenomenon.

\section{APPENDIX B: SPIN OBSERVABLES}

\section{Holstein-Primakoff approximation}

Here we use a Holstein-Primakoff approximation [109] to obtain analytical expressions for spin mean values and fluctuations, which can be linked to the Liouvillian gap in the ferromagnetic phase. The exact HP transformation writes the angular momentum operator in terms of a bosonic mode with annihilation operator $b$ :

$$
S_{-}=\left(\sqrt{2 J-b^{\dagger} b}\right) b, \quad S_{z}=b^{\dagger} b-J .
$$

The HP approximation, consisting of a truncated series expansion of the square root in Eq. (B1), is based on the premise that the upper levels of the finite ladder of eigenstates of $S_{z}$ are not occupied. Therefore, the nonlinear features that distinguish such a finite ladder from the infinite one of a harmonic oscillator are negligible and $S_{-}$is accurately described by the bosonic operator $b$. We will use Eqs. (B1) for $\theta<\pi / 4$ (where we know they are a better description since the system tends to be polarized towards the $-z$ direction) and assume that the same result applies for $\theta>\pi / 4$ by flipping the spin and changing the parameters $\Gamma_{-} \leftrightarrow \Gamma_{-}+$.

Following the approach outlined in Ref. [13], we use a displaced operator

$$
b \rightarrow b+\sqrt{J} \beta
$$

that accounts for the mean polarization of the system. Using the renormalized operators $s_{-} \equiv S_{-} / J$ and $s_{z} \equiv S_{z} / J$, the corresponding HP expression expanded in terms of $\epsilon=1 / \sqrt{J}$ reads

$$
s_{-}=\sqrt{k} \sqrt{1-\epsilon \frac{\beta b^{\dagger}+\beta^{*} b}{k}-\epsilon^{2} \frac{b^{\dagger} b}{k}}(\beta+\epsilon b)=\sum_{i} \epsilon^{i} s_{-}^{(i)},
$$

with $k=2-|\beta|^{2}$. Up to first order in $\epsilon$, we have

$$
\begin{aligned}
& s_{-}^{(0)}=\sqrt{k} \beta, \\
& s_{-}^{(1)}=\frac{1}{2 \sqrt{k}}\left[\left(2 k-|\beta|^{2}\right) b-\beta^{2} b^{\dagger}\right] .
\end{aligned}
$$

For the $s_{z}$ operator, we have $s_{z}=\sum_{i} \epsilon^{i} s_{z}^{(i)}$, with

$$
\begin{aligned}
& s_{z}^{(0)}=|\beta|^{2}-1, \\
& s_{z}^{(1)}=\beta b^{\dagger}+\beta^{*} b .
\end{aligned}
$$

It is useful to expand Eq. (1) as

$$
\begin{aligned}
\dot{\rho}= & -i\left[\Omega S_{x}, \rho\right]+\frac{\Gamma_{-}}{2 J} \mathcal{L}_{S_{-}} \rho+\frac{\Gamma_{+}}{2 J} \mathcal{L}_{S_{+}} \rho \\
& +\frac{\chi}{2 J}\left(2 S_{-} \rho S_{-}-\left\{S_{-}^{2}, \rho\right\}+2 S_{+} \rho S_{+}-\left\{S_{+}^{2}, \rho\right\}\right),
\end{aligned}
$$

where $\Gamma_{ \pm}$are defined by Eqs. (4a) and (4b), $\chi \equiv \Gamma \sin \theta \cos \theta$, and we defined the Lindblad operators $\mathcal{L}_{\mathcal{O}}\{\rho\} \equiv 2 \mathcal{O} \rho \mathcal{O}^{\dagger}-$ $\mathcal{O}^{\dagger} \mathcal{O} \rho-\rho \mathcal{O}^{\dagger} \mathcal{O}$. Then we obtain

$$
\begin{aligned}
\frac{1}{J} \dot{\rho}= & -i\left[\Omega s_{x}, \rho\right]+\frac{\Gamma_{-}}{2} \mathcal{L}_{s_{-}}\{\rho\}+\frac{\Gamma_{+}}{2} \mathcal{L}_{s_{+}}\{\rho\} \\
& +\frac{\chi}{2}\left(2 s_{-} \rho s_{-}-\left\{s_{-}^{2}, \rho\right\}+2 s_{+} \rho s_{+}-\left\{s_{+}^{2}, \rho\right\}\right) \\
= & {\left[\mathcal{L}^{(0)}+\epsilon \mathcal{L}^{(1)}+\epsilon^{2} \mathcal{L}^{(2)}+O\left(\epsilon^{3}\right)\right] \rho . }
\end{aligned}
$$

From Eq. (B5) we immediately obtain $\mathcal{L}^{(0)}=0$. To all orders in the expansion, the Hamiltonian term describing coherent driving can be grouped together with a term coming from the 
dissipative part, in the following form:

$$
-\frac{i}{2}\left\{s_{+}^{(n)}\left[\Omega-i s_{-}^{(0)}\left(\Gamma_{+}-\Gamma_{-}\right)\right]+\text {H.c., } \rho\right\} .
$$

We can therefore simplify the dynamics by eliminating the driving terms to all orders if we choose a proper value for the displacement $\beta$ such that

$$
\Omega-i s_{-}^{(0)}\left(\Gamma_{+}-\Gamma_{-}\right)=\Omega-i \sqrt{2-|\beta|^{2}} \beta\left(\Gamma_{+}-\Gamma_{-}\right)=0 .
$$

This equation has three solutions that, written in terms of $r$ and $\phi$ as $\beta_{i}=r_{i} e^{i \phi_{i}}$, read

$$
\begin{array}{ll}
r_{1}=\sqrt{1+M}, & \phi_{1}=-\pi / 2, \\
r_{2}=\sqrt{1-M}, & \phi_{2}=-\pi / 2, \\
r_{3}=\sqrt{1+Q}, & \phi_{3}=\pi,
\end{array}
$$

where $M$ is given by Eq. (7) and we defined

$$
Q \equiv \sqrt{1+\left(\frac{\Omega}{\Gamma_{+}-\Gamma_{-}}\right)^{2}} .
$$

The first two solutions only exist only when $r_{1}$ and $r_{2}$ are real; we can identify the point at which these solutions cease to exist as the critical point where the phase transition occurs and the HP approximation is not well suited to describe the new phase. The critical lines $\Omega_{c}(\theta)$ that we get in this way coincide with the mean-field result (5), since determining $\beta$ is essentially analogous to determining the steady-state meanfield solution.

We proceed now to demonstrate that $\beta=\beta_{1}$ is the only valid choice for the displacement by analyzing the dynamics of the bosonic mode. Since all the terms of the form (B8) are canceled, we have $\mathcal{L}^{(1)}=0$. We define $A \equiv\left(2 k-|\beta|^{2}\right) / 2 \sqrt{k}$ and $B \equiv-\beta^{2} / 2 \sqrt{k}$, so that $s_{-}^{(1)}=A b+B b^{\dagger}$, and expand the density matrix $\rho(t)=\sum_{n} \epsilon^{n} \rho^{(n)}(t)$. By equating powers of $\epsilon$, Eq. (B7) yields a master equation for the lowest-order density matrix $\rho^{(0)}(t)$,

$$
\begin{aligned}
\dot{\rho}^{(0)}(t)= & \mathcal{L}^{(2)} \rho^{(0)}(t)=\frac{\gamma_{-}}{2} \mathcal{L}_{b}\left\{\rho^{(0)}\right\}+\frac{\gamma_{+}}{2} \mathcal{L}_{b^{\dagger}}\left\{\rho^{(0)}\right\} \\
& +\frac{\eta}{2}\left(2 b \rho^{(0)} b-\left\{b b, \rho^{(0)}\right\}+2 b^{\dagger} \rho^{(0)} b^{\dagger}\right. \\
& \left.-\left\{b^{\dagger} b^{\dagger}, \rho^{(0)}\right\}\right),
\end{aligned}
$$

where $\gamma_{-} \equiv \Gamma_{-} A^{2}+\Gamma_{+} B^{2}+2 \chi A B, \gamma_{+} \equiv \Gamma_{+} A^{2}+\Gamma_{-} B^{2}+$ $2 \chi A B$, and $\eta \equiv A B\left(\Gamma_{-}+\Gamma_{+}\right)+\chi\left(A^{2}+B^{2}\right)$ are all real quantities (since $\beta=-i r_{1}$ is purely imaginary). The dynamics for $\langle b\rangle$ and $\left\langle b^{\dagger}\right\rangle$ is given by the equation $\dot{\mathbf{v}}=\mathcal{W} \mathbf{v}$, with $\mathbf{v}=\left(\langle b\rangle,\left\langle b^{\dagger}\right\rangle\right)^{\top}$ and

$$
\mathcal{W}=\frac{1}{2}\left(\begin{array}{cc}
\gamma_{+}-\gamma_{-} & 0 \\
0 & \gamma_{+}-\gamma_{-}
\end{array}\right)
$$

The eigenvalues of $\mathcal{W}$ describe the energy excitation spectrum of the Liouvillian [13] with the highest real part, to the lowest order in $\epsilon$. We therefore find that the gap in the Liouvillian $\lambda=\left(\gamma_{+}-\gamma_{-}\right) / 2$ is purely real:

$$
\lambda=\frac{\Gamma_{+}-\Gamma_{-}}{2}\left(A^{2}-B^{2}\right)=-\left(\Gamma_{-}-\Gamma_{+}\right)\left(1-|\beta|^{2}\right) .
$$

From the three values of $\beta_{i}=r_{i} e^{i \phi_{i}}$ we get

$$
\begin{aligned}
& \lambda_{1}=\left(\Gamma_{-}-\Gamma_{+}\right) M, \\
& \lambda_{2}=-\left(\Gamma_{-}-\Gamma_{+}\right) M, \\
& \lambda_{3}=\left(\Gamma_{-}-\Gamma_{+}\right) Q .
\end{aligned}
$$

These three solutions are shown in Fig. 11. Only $\lambda_{1}$ has a negative real part in the region $\Omega<\Gamma_{-}-\Gamma_{+}$where these solutions are valid, and therefore the only valid choice of displacement is

$$
\beta=e^{-i \pi / 2} \sqrt{1+M} .
$$

The other choices give $\gamma_{+}>\gamma_{-}$, which clearly yield unstable equations of motion for the bosonic mode, since the effective pumping is larger than the losses and observables diverge; this is related to the instability of the corresponding steady meanfield solutions. The point where the gap closes $\gamma_{+}=\gamma_{-}$is therefore associated with this instability in the equations of motion of the bosonic mode; this indicates that fluctuations in the spin become comparable to $J$ and indicates the onset of the dissipative phase transition.

\section{Spin polarization}

We can now compute spin observables in the ferromagnetic phase, where the HP expansion holds. In order to expand spin mean values $\left\langle s_{z / \pm}\right\rangle$ in powers of $\epsilon$, we must take into account both the HP expansions [Eqs. (B4) and (B5)] and the expansion of $\rho(t)$. Doing so, we obtain, to order $\epsilon^{2}$,

$$
\begin{aligned}
\left\langle s_{z / \pm}(t)\right\rangle= & \operatorname{Tr}\left[s_{z / \pm}^{(0)} \rho^{(0)}\right]+\epsilon\left\{\operatorname{Tr}\left[s_{z / \pm}^{(1)} \rho^{(0)}\right]+\operatorname{Tr}\left[s_{z / \pm}^{(0)} \rho^{(1)}\right]\right\} \\
& +\epsilon^{2}\left\{\operatorname{Tr}\left[s_{z / \pm}^{(2)} \rho^{(0)}\right]+\operatorname{Tr}\left[s_{z / \pm}^{(1)} \rho^{(1)}\right]\right. \\
& \left.+\operatorname{Tr}\left[s_{z / \pm}^{(0)} \rho^{(2)}\right]\right\}+O\left(\epsilon^{3}\right),
\end{aligned}
$$

where we omitted the time dependence of the $\rho^{(n)}(t)$ for simplicity. Noting that $s_{z / \pm}^{(0)}$ is a c-number and that, by definition, $\operatorname{Tr}\left[\rho^{(1)}\right]=\operatorname{Tr}\left[\rho^{(2)}\right]=0$, the terms $\operatorname{Tr}\left[s_{z / \pm}^{(0)} \rho^{(1)}\right]$ and $\operatorname{Tr}\left[s_{z / \pm}^{(0)} \rho^{(2)}\right]$ in Eq. (B17) are equal to zero.

However, there are nonvanishing terms proportional to $\epsilon^{2}$ that depend on $\rho^{(1)}$. Since the effective master equation $\dot{\rho}^{(1)}(t)=\mathcal{L}^{(2)} \rho^{(1)}+\mathcal{L}^{(3)} \rho^{(0)}$ is no longer quadratic, these terms prevent us from obtaining a closed expression for $\left\langle s_{z / \pm}\right\rangle$ at order $\epsilon^{2}=1 / J$.

Let us define the correlators to zeroth order in $\rho$ as $\langle\mathcal{O}\rangle_{0} \equiv$ $\operatorname{Tr}\left[\mathcal{O} \rho_{0}\right]$. In order to evaluate the first-order terms $\left\langle s_{z / \pm}^{(1)}\right\rangle_{0}$ in Eq. (B17), we must use Eq. (B12) to obtain correlators of the form $\langle b\rangle_{0}$. In general, the dynamics of any arbitrary correlator $\left\langle b^{\dagger m} b^{n}\right\rangle_{0}$ will be given by

$$
\begin{aligned}
\frac{d\left\langle b^{\dagger m} b^{n}\right\rangle_{0}}{d t}= & \frac{\gamma_{+}-\gamma_{-}}{2}(n+m)\left\langle b^{\dagger^{\dagger}} b^{n}\right\rangle_{0} \\
& +\gamma_{+} m n\left\langle b^{\dagger^{m-1}} b^{n-1}\right\rangle_{0}-\frac{\eta}{2} m(m-1)\left\langle b^{\dagger^{m-2}} b^{n}\right\rangle_{0} \\
& -\frac{\eta}{2} n(n-1)\left\langle b^{\dagger^{m}} b^{n-2}\right\rangle_{0} .
\end{aligned}
$$

In particular, we are interested in the stationary limit $t \rightarrow \infty$, where the density matrix fulfills $\mathcal{L}^{(2)} \rho^{(0)}=0$ (in the following, the notation \langle\rangle$_{0}$ and $\rho^{(n)}$ will refer to stationary values). 
We obtain steady-state values of the correlators by setting the derivatives of Eq. (B18) equal to zero. This way, we get, for the case $m=0$ and $n=1$,

$$
\langle b\rangle_{0}=0 .
$$

Since $\left\langle s_{z}^{(1)}\right\rangle_{0}$ and $\left\langle s_{ \pm}^{(1)}\right\rangle_{0}$ are proportional to $\langle b\rangle_{0}$ and $\left\langle b^{\dagger}\right\rangle_{0}$, we find that they are all zero and therefore conclude that $\left\langle s_{z / \pm}\right\rangle$ has no first-order dependence on $\epsilon$. Therefore, using Eqs. (B5), (B4), and (B16), we find that the stationary expectation values $\left\langle s_{z}\right\rangle,\left\langle s_{x}\right\rangle$, and $\left\langle s_{y}\right\rangle$ are given, with corrections to second order in $\epsilon$, by the zeroth-order terms $\operatorname{Tr}\left[s_{z / \pm}^{(0)} \rho^{(0)}\right]$, which coincide with the solutions of the mean-field equations (A5):

$$
\begin{aligned}
& \left\langle s_{z}\right\rangle=M=-R+O\left(\epsilon^{2}\right), \\
& \left\langle s_{x}\right\rangle=0+O\left(\epsilon^{2}\right), \\
& \left\langle s_{y}\right\rangle=\frac{\Omega}{\Gamma_{+}-\Gamma_{-}}+O\left(\epsilon^{2}\right) .
\end{aligned}
$$

\section{Spin fluctuations: Spin squeezing}

Our lack of an analytical expression for $\rho^{(n)}$ for $n>0$ prevents us from obtaining closed-form expressions for the second-order corrections to the mean spin. However, it is possible to get expressions for the fluctuations $\Delta s_{z / \pm}^{2}$ to second order, which is the lowest in their expansion. In particular, it is easy to prove that

$$
\Delta s_{z / \pm}^{2}=\left\langle s_{z / \pm}^{(1)}\right\rangle_{0}^{2}+O\left(\epsilon^{3}\right)
$$

The mean spin direction in the thermodynamic limit, obtained from Eqs. (B20a)-(B20c), can be written as

$$
\begin{aligned}
\mathbf{u}_{m} & =\frac{\left\langle s_{x}\right\rangle \mathbf{u}_{x}+\left\langle s_{y}\right\rangle \mathbf{u}_{y}+\left\langle s_{z}\right\rangle \mathbf{u}_{z}}{\sqrt{\left\langle\mathbf{s}^{2}\right\rangle}} \\
& =\sqrt{1-M^{2}} \mathbf{u}_{y}+M \mathbf{u}_{z} .
\end{aligned}
$$

We are interested in the squeezing along some direction in the plane perpendicular to $\mathbf{u}_{m}, \mathbf{u}_{\perp}(\varphi) \equiv \cos (\varphi) \mathbf{u}_{x}-$ $\sin (\varphi)\left[-M \mathbf{u}_{y}+\sqrt{1-M^{2}} \mathbf{u}_{z}\right]$; this direction is to be determined by finding the $\varphi$ that maximizes the squeezing. As we prove in Appendix B 4, $\mathbf{u}_{x}$ is always the preferential direction of squeezing. In order to compute $\xi_{\perp}$, it is useful to obtain, from the solution of Eq. (B12), the expression for the mean quadratic correlators

$$
\begin{aligned}
\left\langle b^{\dagger} b\right\rangle & =\frac{\gamma_{+}}{\gamma_{-}-\gamma_{+}}, \\
\left\langle b^{2}\right\rangle & =\left\langle b^{\dagger}{ }^{2}\right\rangle=\frac{\eta}{\gamma_{+}-\gamma_{-}} .
\end{aligned}
$$

Using these, we can write the expression for the variance

$$
\Delta s_{x}^{2}=\frac{k}{2 J}\left(\left\langle b^{\dagger} b\right\rangle-\left\langle b^{2}\right\rangle+\frac{1}{2}\right)+O\left(\epsilon^{3}\right)
$$

and from there obtain the expression for the spin squeezing

$$
\xi_{\perp}^{2}=\frac{N\left(\Delta S_{x}\right)^{2}}{\langle\mathbf{S}\rangle^{2}}=k\left(\frac{\gamma_{+}-\eta}{\gamma_{-}-\gamma_{+}}+\frac{1}{2}\right)+O(\epsilon)
$$

which can be rewritten in the form shown in Eq. (9).

\section{Preferential direction of squeezing}

To complete our previous discussion, we demonstrate here that $\mathbf{u}_{x}$ is the direction with minimum fluctuations finding the angle $\varphi$ that minimizes spin fluctuations along the general direction $\mathbf{u}_{\perp}(\varphi)$. We define the short notation for the quantities with the properties of sine and cosines, $c \equiv \cos (\varphi)$, $s \equiv \sin (\varphi), \tilde{c} \equiv M$, and $\tilde{s} \equiv \sqrt{1-M^{2}}$, and define the covariance $\operatorname{cov}[X, Y] \equiv\langle(X-\langle X\rangle)(Y-\langle Y\rangle)\rangle$. Then we get, for the fluctuations along a general direction perpendicular to the mean spin,

$$
\begin{aligned}
\frac{\left(\Delta S_{\perp}\right)^{2}}{J^{2}}= & \frac{1}{J^{2}}\left\{s^{2}\left[\tilde{c}^{2} \frac{2 \operatorname{cov}\left[S_{+}, S_{-}\right]-\left(\Delta S_{+}\right)^{2}-\left(\Delta S_{-}\right)^{2}-2\left\langle S_{z}\right\rangle}{4}+\tilde{s}^{2}\left(\Delta S_{z}\right)^{2}-i \tilde{s} \tilde{c}\left(\left(\Delta S_{+} S_{z}\right)^{2}-\left(\Delta S_{-} S_{z}\right)^{2}+\frac{\left\langle S_{+}\right\rangle+\left\langle S_{-}\right\rangle}{2}\right)\right]\right. \\
& +c^{2}\left[\frac{2 \operatorname{cov}\left[S_{+}, S_{-}\right]+\left(\Delta S_{+}\right)^{2}+\left(\Delta S_{-}\right)^{2}-2\left\langle S_{z}\right\rangle}{4}\right] \\
& \left.+s c\left[i \tilde{c} \frac{\left(\Delta S_{+}\right)^{2}-\left(\Delta S_{-}\right)^{2}}{2}-\tilde{s}\left(\operatorname{cov}\left[S_{+}, S_{z}\right]+\operatorname{cov}\left[S_{-}, S_{z}\right]+\frac{\left\langle S_{+}\right\rangle-\left\langle S_{-}\right\rangle}{2}\right)\right]\right\},
\end{aligned}
$$

which we can express, grouping the coefficients of $s^{2}, c^{2}$, and $s c$ into three parameters $\kappa, \lambda$, and $\mu$, respectively, as

$$
\frac{\left(\Delta S_{\perp}\right)^{2}}{J^{2}}=\frac{1}{J}\left[\kappa \sin (\varphi)^{2}+\lambda \cos (\varphi)^{2}+\mu \cos (\varphi) \sin (\varphi)\right]=\frac{1}{2 J}[(\lambda-\kappa) \cos (2 \varphi)+\mu \sin (2 \varphi)+\kappa+\lambda] .
$$

To find the angle $\varphi$ that minimizes $\left(\Delta S_{\perp}\right)^{2}$, we take the derivative with respect to $\varphi$ and make it equal to zero, giving the following solution for $\varphi$ :

$$
2 \varphi=\arctan \left(\frac{\mu}{\lambda-\kappa}\right)
$$

This function is usually treated as a single-valued function by restricting the domain of $\tan (x)$ to $x \in[-\pi / 2, \pi / 2]$. We know from numerical calculations that indeed $\varphi \approx 0$, so we use this single-valued definition of $\arctan (x)$. In that case, we can use the properties

$$
\begin{aligned}
& \cos [\arctan (x)]=\frac{1}{\sqrt{x^{2}+1}}, \\
& \sin [\arctan (x)]=\frac{x}{\sqrt{x^{2}+1}}
\end{aligned}
$$



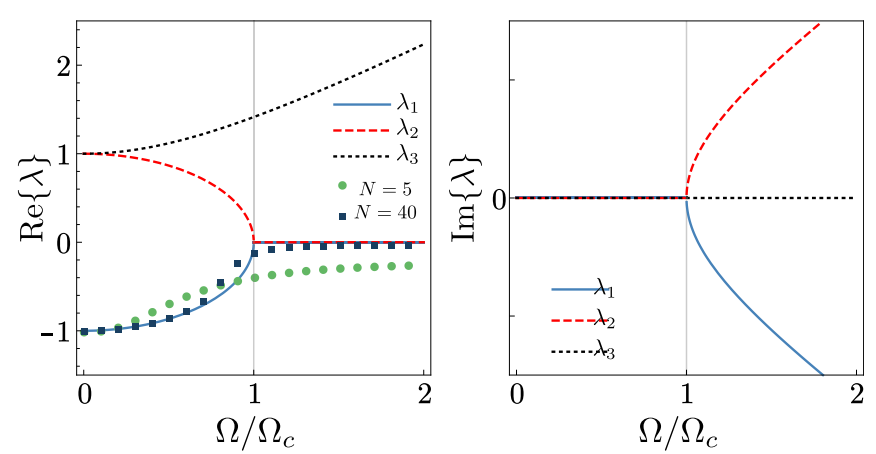

FIG. 11. Eigenvalues as a function of the normalized driving amplitude $\Omega /\left(\Gamma_{-}-\Gamma_{+}\right)$, assuming $\Gamma_{-}>\Gamma_{+}$. Lines shows the analytical solutions given by Eq. (B15). Markers indicate the numerical solutions for finite systems. Below $\Omega /\left(\Gamma_{-}-\Gamma_{+}\right)=1$, the only valid solution is $\lambda_{1}$.

and then get

$$
\frac{\left(\Delta S_{\perp}\right)^{2}}{J^{2}}=\frac{1}{2 J}\left[\kappa+\lambda+(\lambda-\kappa) \sqrt{1+\left(\frac{\mu}{\lambda-\kappa}\right)^{2}}\right] .
$$

We are now left to compute the values of $\kappa, \lambda$, and $\mu$. To do so, let us observe that, to order $\epsilon^{2}$,

$$
\frac{\left\langle S_{-}^{2}\right\rangle}{J^{2}}=\left\langle s_{-}^{(0)^{2}}\right\rangle+\frac{1}{J}\left[\left\langle s_{-}^{(1)^{2}}\right\rangle+\left\langle s_{-}^{(0)} s_{-}^{(2)}\right\rangle+\left\langle s_{-}^{(2)} s_{-}^{(0)}\right\rangle\right],
$$

and since $s_{-}^{(0)}$ is a c-number, we have that $\left(\Delta S_{-}\right)^{2} / J^{2}=$ $\left\langle s_{-}^{(1)^{2}}\right\rangle / J$. By following the same argument to express the rest of the variances and covariances present in the equation in terms of the $s_{ \pm, z}^{(n)}$, we can write down the following values of $\kappa, \lambda$, and $\mu$, to zeroth order in $\epsilon$ :

$$
\begin{aligned}
\kappa= & \tilde{c}^{2} \frac{2\left\langle s_{+}^{(1)} s_{-}^{(1)}\right\rangle-\left\langle s_{+}^{(1)^{2}}\right\rangle-\left\langle s_{-}^{(1)^{2}}\right\rangle-2\left\langle s_{z}^{(0)}\right\rangle}{4}+\tilde{s}^{2}\left\langle s_{z}^{(1)^{2}}\right\rangle \\
- & i \tilde{s} \tilde{c}\left[\left\langle s_{+}^{(1)} s_{z}^{(1)}\right\rangle-\left\langle s_{-}^{(1)} s_{z}^{(1)}\right\rangle+\frac{\left\langle s_{+}^{(0)}\right\rangle+\left\langle s_{-}^{(0)}\right\rangle}{2}\right], \\
\lambda & =\frac{2\left\langle s_{+}^{(1)} s_{-}^{(1)}\right\rangle+\left\langle s_{+}^{(1)^{2}}\right\rangle+\left\langle s_{-}^{(1)^{2}}\right\rangle-2\left\langle s_{z}^{(0)}\right\rangle}{4}, \\
\mu= & i \tilde{c} \frac{\left\langle s_{+}^{(1)^{2}}\right\rangle-\left\langle s_{-}^{(1)^{2}}\right\rangle}{2} \\
& -\tilde{s}\left(\left\langle s_{+}^{(1)} s_{z}^{(1)}\right\rangle+\left\langle s_{-}^{(1)} s_{z}^{(1)}\right\rangle+\frac{\left\langle s_{+}^{(0)}\right\rangle-\left\langle s_{-}^{(0)}\right\rangle}{2}\right) .
\end{aligned}
$$

Taking into account that $\left\langle b^{2}\right\rangle=\left\langle b^{\dagger^{2}}\right\rangle$ and $s_{-}^{(1)}=A b+B b^{\dagger}$, we can write the expressions of the correlators appearing in the equations:

$$
\begin{aligned}
\left\langle s_{ \pm}^{(1)^{2}}\right\rangle & =\left\langle b^{2}\right\rangle\left(A^{2}+B^{2}\right)+2 A B\left\langle b^{\dagger} b\right\rangle+A B, \\
\left\langle s_{+}^{(1)} s_{-}^{(1)}\right\rangle & =\left\langle b^{\dagger} b\right\rangle\left(A^{2}+B^{2}\right)+\left\langle b^{2}\right\rangle 2 A B+B^{2}, \\
\left\langle s_{z}^{(1)^{2}}\right\rangle & =|\beta|^{2}\left[2\left(\left\langle b^{\dagger} b\right\rangle-\left\langle b^{2}\right\rangle\right)+1\right], \\
\left\langle s_{+}^{(1)} s_{z}^{(1)}\right\rangle & =i|\beta|(A-B)\left(\left\langle b^{\dagger} b\right\rangle-\left\langle b^{2}\right\rangle\right)+B \beta, \\
\left\langle s_{-}^{(1)} s_{z}^{(1)}\right\rangle & =i|\beta|(A-B)\left(\left\langle b^{2}\right\rangle-\left\langle b^{\dagger} b\right\rangle\right)+A \beta .
\end{aligned}
$$

We know that $\left\langle s_{-}^{(0)}\right\rangle=\sqrt{k} \beta=-\left\langle s_{+}^{(0)}\right\rangle$, and from Eqs. (B35e) and (B35e) we have that $\left\langle s_{+}^{(1)} s_{z}^{(1)}\right\rangle+\left\langle s_{-}^{(1)} s_{z}^{(1)}\right\rangle=\beta(A+B)=$ $-i|\beta| \sqrt{k}=-i \tilde{s}$. Also, $\left\langle s_{+}^{(1)^{2}}\right\rangle=\left\langle s_{-}^{(1)^{2}}\right\rangle$. It is then easy to see that

$$
\mu=0 \rightarrow \varphi=0,
$$

proving that, in the thermodynamic limit, $\mathbf{u}_{x}$ is always the preferential direction for squeezing.

\section{APPENDIX C: PROBABILITY AMPLITUDES OF GENERAL MONTE CARLO TRAJECTORIES}

In this Appendix we demonstrate Eq. (21). By expanding the wave function in eigenstates $|m\rangle$ of the strong symmetry, we find, for a trajectory with jumps at times $\left(t_{1}, \ldots, t_{n}\right)<t$,

$$
\begin{aligned}
|\psi(t)\rangle & \propto e^{-i \tilde{H}\left(t-t_{n}\right)} A\left|\psi\left(T_{n}\right)\right\rangle \propto \sum_{m} e^{-i \tilde{H}\left(t-t_{n}\right)} m c_{m}\left(t_{n}\right)|m\rangle \\
& \propto \sum_{m} e^{-i \tilde{H}\left(t-t_{n}\right)} m e^{-i \tilde{H}\left(t_{n}-t_{n-1}\right)} m c_{m}\left(t_{n-1}\right)|m\rangle \\
& \propto \cdots \propto e^{-i \tilde{H} t} m^{n} c_{m}(0)|m\rangle .
\end{aligned}
$$

From here, taking into account that $\tilde{H}=H-i \Gamma A^{\dagger} A / 2 J$, the probability to find the $|\psi(t)\rangle$ in an eigenstate $|m\rangle$ simply reads

$$
p(m ; t, n)=|\langle m \mid \psi(t)\rangle|^{2}=\frac{1}{\mathcal{N}_{t, n}} e^{-\Gamma|m|^{2} t / J}|m|^{2 n}\left|c_{m}(0)\right|^{2},
$$

with $\mathcal{N}_{t, n}$ a normalization constant. Defining a rate $\alpha=$ $n J / \Gamma t$, we can rewrite Eq. (C2) as

$$
p(m ; t, \alpha)=\frac{1}{\mathcal{N}_{t, \alpha}}\left(e^{-|m|^{2}}|m|^{2 \alpha}\right)^{t \Gamma / J}\left|c_{m}(0)\right|^{2} .
$$

\section{APPENDIX D: EXACT EXPRESSION FOR THE ACTIVITY DISTRIBUTION}

In this Appendix we demonstrate Eq. (23). Defining the quantum-jump superoperator $\mathcal{J}\{\cdot\} \equiv L\{\cdot\} L^{\dagger}$ and the no-jump part of the Liouvillian $\mathcal{S}=\mathcal{L}-\mathcal{J}$, the probability for the system to experience $K$ quantum jumps on a time $T$, starting at the state $\rho(0)$, is given by $[38,110]$

$$
\begin{aligned}
p_{T}(K)= & \int_{0}^{T} d t_{K} \int_{0}^{t_{K}} d t_{K-1} \cdots \int_{0}^{t_{2}} d t_{1} \\
& \times \operatorname{Tr}\left[e^{\mathcal{S}\left(T-t_{K}\right)} \mathcal{J} e^{\mathcal{S}\left(t_{K}-t_{K-1}\right)} \cdots \mathcal{J} e^{\mathcal{S} t_{1}} \rho(0)\right] .
\end{aligned}
$$

From Eqs. (11a) and (11b) we find

$$
\begin{aligned}
e^{\mathcal{S} t}|m\rangle\langle m| & =e^{-\Gamma|m|^{2} t / J}|m\rangle\langle m|, \\
\mathcal{J}|m\rangle\langle m| & =\frac{\Gamma}{J}|m|^{2}|m\rangle\langle m|,
\end{aligned}
$$

and therefore

$$
\begin{aligned}
p_{T}(K) & =\int_{0}^{T} d t_{K} \cdots \int_{0}^{t_{2}} d t_{1} \sum_{m} c_{m}\left(\frac{\Gamma}{J}|m|^{2}\right)^{K} e^{-\Gamma|m|^{2} T / J} \\
& =\sum_{m} \frac{1}{K !}\left(\frac{T \Gamma|m|^{2}}{J}\right)^{K} e^{-\Gamma|m|^{2} T / J} c_{m} .
\end{aligned}
$$


[1] A. Amo, D. Sanvitto, F. P. Laussy, D. Ballarini, E. del Valle, M. D. Martin, A. Lemaître, J. Bloch, D. N. Krizhanovskii, M. S. Skolnick, C. Tejedor, and L. Viña, Collective fluid dynamics of a polariton condensate in a semiconductor microcavity, Nature (London) 457, 291 (2009).

[2] S. R. K. Rodriguez, W. Casteels, F. Storme, N. Carlon Zambon, I. Sagnes, L. Le Gratiet, E. Galopin, A. Lemaître, A. Amo, C. Ciuti, and J. Bloch, Probing a Dissipative Phase Transition via Dynamical Optical Hysteresis, Phys. Rev. Lett. 118, 247402 (2017).

[3] T. Fink, A. Schade, S. Höfling, C. Schneider, and A. Imamoglu, Signatures of a dissipative phase transition in photon correlation measurements, Nat. Phys. 14, 365 (2018).

[4] C. Carr, R. Ritter, C. G. Wade, C. S. Adams, and K. J. Weatherill, Nonequilibrium Phase Transition in a Dilute Rydberg Ensemble, Phys. Rev. Lett. 111, 113901 (2013).

[5] N. R. de Melo, C. G. Wade, N. Šibalić, J. M. Kondo, C. S. Adams, and K. J. Weatherill, Intrinsic optical bistability in a strongly driven Rydberg ensemble, Phys. Rev. A 93, 063863 (2016).

[6] M. Fitzpatrick, N. M. Sundaresan, A. C. Y. Li, J. Koch, and A. A. Houck, Observation of a Dissipative Phase Transition in a One-Dimensional Circuit QED Lattice, Phys. Rev. X 7, 011016 (2017).

[7] K. Baumann, C. Guerlin, F. Brennecke, and T. Esslinger, Dicke quantum phase transition with a superfluid gas in an optical cavity, Nature (London) 464, 1301 (2010).

[8] J. Klinder, H. Keßler, M. Wolke, L. Mathey, and A. Hemmerich, Dynamical phase transition in the open Dicke model, Proc. Natl. Acad. Sci. USA 112, 3290 (2015).

[9] C. Hamsen, K. N. Tolazzi, T. Wilk, and G. Rempe, Strong coupling between photons of two light fields mediated by one atom, Nat. Phys. 14, 885 (2018).

[10] J. Teufel, T. Donner, D. Li, J. Harlow, M. Allman, K. Cicak, A. Sirois, J. D. Whittaker, K. Lehnert, and R. W. Simmonds, Sideband cooling of micromechanical motion to the quantum ground state, Nature (London) 475, 359 (2011).

[11] S. Kolkowitz, A. C. B. Jayich, Q. P. Unterreithmeier, S. D. Bennett, P. Rabl, J. Harris, and M. D. Lukin, Coherent sensing of a mechanical resonator with a single-spin qubit, Science 335, 1603 (2012).

[12] B. Pigeau, S. Rohr, L. M. De Leípinay, A. Gloppe, V. Jacques, and O. Arcizet, Observation of a phononic Mollow triplet in a multimode hybrid spin-nanomechanical system, Nat. Commun. 6, 8603 (2015).

[13] E. M. Kessler, G. Giedke, A. Imamoglu, S. F. Yelin, M. D. Lukin, and J. I. Cirac, Dissipative phase transition in a central spin system, Phys. Rev. A 86, 012116 (2012).

[14] F. Minganti, A. Biella, N. Bartolo, and C. Ciuti, Spectral theory of Liouvillians for dissipative phase transitions, Phys. Rev. A 98, 042118 (2018).

[15] H. J. Carmichael, Breakdown of Photon Blockade: A Dissipative Quantum Phase Transition in Zero Dimensions, Phys. Rev. X 5, 031028 (2015).

[16] H. Weimer, Variational Principle for Steady States of Dissipative Quantum Many-Body Systems, Phys. Rev. Lett. 114, 040402 (2015).

[17] M. Benito, C. Sánchez Muñoz, and C. Navarrete-Benlloch, Degenerate parametric oscillation in quantum membrane optomechanics, Phys. Rev. A 93, 023846 (2016).
[18] L. M. Sieberer, S. D. Huber, E. Altman, and S. Diehl, Dynamical Critical Phenomena in Driven-Dissipative Systems, Phys. Rev. Lett. 110, 195301 (2013).

[19] C. Sánchez Muñoz, A. Lara, J. Puebla, and F. Nori, Hybrid Systems for the Generation of Nonclassical Mechanical States via Quadratic Interactions, Phys. Rev. Lett. 121, 123604 (2018).

[20] M. Biondi, G. Blatter, H. E. Türeci, and S. Schmidt, Nonequilibrium gas-liquid transition in the driven-dissipative photonic lattice, Phys. Rev. A 96, 043809 (2017).

[21] M.-J. Hwang, P. Rabl, and M. B. Plenio, Dissipative phase transition in the open quantum Rabi model, Phys. Rev. A 97, 013825 (2018).

[22] J. J. Mendoza-Arenas, S. R. Clark, S. Felicetti, G. Romero, E. Solano, D. G. Angelakis, and D. Jaksch, Beyond meanfield bistability in driven-dissipative lattices: Bunchingantibunching transition and quantum simulation, Phys. Rev. A 93, 023821 (2016).

[23] J. S. Ferreira and P. Ribeiro, The Lipkin-Meshkov-Glick model with Markovian dissipation-A description of a collective spin on a metallic surface, arXiv:1801.00818.

[24] K. Macieszczak, M. Guţă, I. Lesanovsky, and J. P. Garrahan, Dynamical phase transitions as a resource for quantum enhanced metrology, Phys. Rev. A 93, 022103 (2016).

[25] S. Fernández-Lorenzo and D. Porras, Quantum sensing close to a dissipative phase transition: Symmetry breaking and criticality as metrological resources, Phys. Rev. A 96, 013817 (2017).

[26] F. Letscher, O. Thomas, T. Niederprüm, M. Fleischhauer, and H. Ott, Bistability versus Metastability in Driven Dissipative Rydberg Gases, Phys. Rev. X 7, 021020 (2017).

[27] P. R. Muppalla, O. Gargiulo, S. I. Mirzaei, B. P. Venkatesh, M. L. Juan, L. Grünhaupt, I. M. Pop, and G. Kirchmair, Bistability in a mesoscopic Josephson junction array resonator, Phys. Rev. B 97, 024518 (2018).

[28] M. J. A. Schuetz, E. M. Kessler, L. M. K. Vandersypen, J. I. Cirac, and G. Giedke, Steady-State Entanglement in the Nuclear Spin Dynamics of a Double Quantum Dot, Phys. Rev. Lett. 111, 246802 (2013).

[29] M. J. A. Schuetz, E. M. Kessler, L. M. K. Vandersypen, J. I. Cirac, and G. Giedke, Nuclear spin dynamics in double quantum dots: Multistability, dynamical polarization, criticality, and entanglement, Phys. Rev. B 89, 195310 (2014).

[30] L. Hruby, N. Dogra, M. Landini, T. Donner, and T. Esslinger, Metastability and avalanche dynamics in strongly correlated gases with long-range interactions, Proc. Natl. Acad. Sci. USA 115, 3279 (2018).

[31] T. E. Lee, H. Häffner, and M. C. Cross, Collective Quantum Jumps of Rydberg Atoms, Phys. Rev. Lett. 108, 023602 (2012).

[32] N. Malossi, M. M. Valado, S. Scotto, P. Huillery, P. Pillet, D. Ciampini, E. Arimondo, and O. Morsch, Full Counting Statistics and Phase Diagram of a Dissipative Rydberg Gas, Phys. Rev. Lett. 113, 023006 (2014).

[33] C. Ates, B. Olmos, J. P. Garrahan, and I. Lesanovsky, Dynamical phases and intermittency of the dissipative quantum Ising model, Phys. Rev. A 85, 043620 (2012).

[34] K. Macieszczak, M. Guţă, I. Lesanovsky, and J. P. Garrahan, Towards a Theory of Metastability in Open Quantum Dynamics, Phys. Rev. Lett. 116, 240404 (2016). 
[35] D. Manzano and P. I. Hurtado, Symmetry and the thermodynamics of currents in open quantum systems, Phys. Rev. B 90, 125138 (2014).

[36] H. Wilming, M. J. Kastoryano, A. H. Werner, and J. Eisert, Emergence of spontaneous symmetry breaking in dissipative lattice systems, J. Math. Phys. 58, 033302 (2017).

[37] J. Hannukainen and J. Larson, Dissipation-driven quantum phase transitions and symmetry breaking, Phys. Rev. A 98, 042113 (2018).

[38] P. Zoller, M. Marte, and D. F. Walls, Quantum jumps in atomic systems, Phys. Rev. A 35, 198 (1987).

[39] J. Dalibard, Y. Castin, and K. Mølmer, Wave-Function Approach to Dissipative Processes in Quantum Optics, Phys. Rev. Lett. 68, 580 (1992).

[40] M. B. Plenio and P. L. Knight, The quantum-jump approach to dissipative dynamics in quantum optics, Rev. Mod. Phys. 70, 101 (1998).

[41] C. Flindt and J. P. Garrahan, Trajectory Phase Transitions, Lee-Yang Zeros, and High-Order Cumulants in Full Counting Statistics, Phys. Rev. Lett. 110, 050601 (2013).

[42] J. M. Hickey, C. Flindt, and J. P. Garrahan, Intermittency and dynamical Lee-Yang zeros of open quantum systems, Phys. Rev. E 90, 062128 (2014).

[43] B. Buča and T. Prosen, A note on symmetry reductions of the Lindblad equation: Transport in constrained open spin chains, New J. Phys. 14, 073007 (2012).

[44] V. V. Albert and L. Jiang, Symmetries and conserved quantities in Lindblad master equations, Phys. Rev. A 89, 022118 (2014).

[45] T. Benoist, C. Pellegrini, and F. Ticozzi, Exponential stability of subspaces for quantum stochastic master equations, Ann. Henri Poincaré 18, 2045 (2017).

[46] T. Benoist and C. Pellegrini, Large time behavior and convergence rate for quantum filters under standard non demolition conditions, Commun. Math. Phys. 331, 703 (2014).

[47] M. van Horssen, Large deviations and dynamical phase transitions for quantum Markov processes, Ph.D. thesis, University of Nottingham, 2014.

[48] D. Jaksch, S. A. Gardiner, K. Schulze, J. I. Cirac, and P. Zoller, Uniting Bose-Einstein Condensates in Optical Resonators, Phys. Rev. Lett. 86, 4733 (2001).

[49] A. Micheli, D. Jaksch, J. I. Cirac, and P. Zoller, Many-particle entanglement in two-component Bose-Einstein condensates, Phys. Rev. A 67, 013607 (2003).

[50] F. Dimer, B. Estienne, A. S. Parkins, and H. J. Carmichael, Proposed realization of the Dicke-model quantum phase transition in an optical cavity QED system, Phys. Rev. A 75, 013804 (2007).

[51] E. G. Dalla Torre, J. Otterbach, E. Demler, V. Vuletic, and M. D. Lukin, Dissipative Preparation of Spin Squeezed Atomic Ensembles in a Steady State, Phys. Rev. Lett. 110, 120402 (2013).

[52] A. Gonzalez-Tudela and D. Porras, Mesoscopic Entanglement Induced by Spontaneous Emission in Solid-State Quantum Optics, Phys. Rev. Lett. 110, 080502 (2013).

[53] B. Buča, J. Tindall, and D. Jaksch, Non-stationary coherent quantum many-body dynamics through dissipation, Nat. Commun. 10, 1730 (2019).

[54] F. Iemini, A. Russomanno, J. Keeling, M. Schirò, M. Dalmonte, and R. Fazio, Boundary Time Crystals, Phys. Rev. Lett. 121, 035301 (2018).
[55] K. Tucker, B. Zhu, R. Lewis-Swan, J. Marino, F. Jimenez, J. Restrepo, and A. M. Rey, Shattered time: Can a dissipative time crystal survive many-body correlations? New J. Phys. 20, 123003 (2018).

[56] J. Tindall, C. Sánchez Munoz, B. Buca, and D. Jaksch, Quantum synchronisation enabled by dynamical symmetries and dissipation, arXiv:1907.12837.

[57] K. Hepp and E. H. Lieb, On the superradiant phase transition for molecules in a quantized radiation field: The Dicke maser model, Ann. Phys. (NY) 76, 360 (1973).

[58] K. Hepp and E. H. Lieb, Equilibrium statistical mechanics of matter interacting with the quantized radiation field, Phys. Rev. A 8, 2517 (1973).

[59] Y. K. Wang and F. T. Hioe, Phase transition in the Dicke model of superradiance, Phys. Rev. A 7, 831 (1973).

[60] C. Emary and T. Brandes, Quantum Chaos Triggered by Precursors of a Quantum Phase Transition: The Dicke Model, Phys. Rev. Lett. 90, 044101 (2003).

[61] H. Keßler, J. Klinder, M. Wolke, and A. Hemmerich, Steering Matter Wave Superradiance with an Ultranarrow-Band Optical Cavity, Phys. Rev. Lett. 113, 070404 (2014).

[62] R. M. Kroeze, Y. Guo, V. D. Vaidya, J. Keeling, and B. L. Lev, Spinor Self-Ordering of a Quantum Gas in a Cavity, Phys. Rev. Lett. 121, 163601 (2018).

[63] M. P. Baden, K. J. Arnold, A. L. Grimsmo, S. Parkins, and M. D. Barrett, Realization of the Dicke Model Using CavityAssisted Raman Transitions, Phys. Rev. Lett. 113, 020408 (2014).

[64] Z. Zhiqiang, C. H. Lee, R. Kumar, K. Arnold, S. J. Masson, A. Parkins, and M. Barrett, Nonequilibrium phase transition in a spin-1 Dicke model, Optica 4, 424 (2017).

[65] J. Keeling, M. J. Bhaseen, and B. D. Simons, Collective Dynamics of Bose-Einstein Condensates in Optical Cavities, Phys. Rev. Lett. 105, 043001 (2010).

[66] M. J. Bhaseen, J. Mayoh, B. D. Simons, and J. Keeling, Dynamics of nonequilibrium Dicke models, Phys. Rev. A 85, 013817 (2012).

[67] D. F. Walls and G. J. Milburn, Quantum Optics (Springer, Berlin, 1994).

[68] R. Puri and S. Lawande, Exact steady-state density operator for a collective atomic system in an external field, Phys. Lett. 72, 200 (1979).

[69] S. Lawande, R. Puri, and S. Hassan, Non-resonant effects in the fluorescent Dicke model. I. Exact steady state analysis, J. Phys. B. 14, 4171 (1981).

[70] G. S. Agarwal, Relation between atomic coherent-state representation, state multipoles, and generalized phase-space distributions, Phys. Rev. A 24, 2889 (1981).

[71] J. P. Dowling, G. S. Agarwal, and W. P. Schleich, Wigner distribution of a general angular-momentum state: Applications to a collection of two-level atoms, Phys. Rev. A 49, 4101 (1994).

[72] L. Pezzè, A. Smerzi, M. K. Oberthaler, R. Schmied, and P. Treutlein, Quantum metrology with nonclassical states of atomic ensembles, Rev. Mod. Phys. 90, 035005 (2018).

[73] C. Gross, T. Zibold, E. Nicklas, J. Esteve, and M. K. Oberthaler, Nonlinear atom interferometer surpasses classical precision limit, Nature (London) 464, 1165 (2010). 
[74] T. Berrada, S. van Frank, R. Bücker, T. Schumm, J.-F. Schaff, and J. Schmiedmayer, Integrated Mach-Zehnder interferometer for Bose-Einstein condensates, Nat. Commun. 4, 2077 (2013).

[75] J. Borregaard and A. S. Sørensen, Near-Heisenberg-Limited Atomic Clocks in the Presence of Decoherence, Phys. Rev. Lett. 111, 090801 (2013).

[76] D. J. Wineland, J. J. Bollinger, W. M. Itano, and D. J. Heinzen, Squeezed atomic states and projection noise in spectroscopy, Phys. Rev. A 50, 67 (1994).

[77] J. Ma, X. Wang, C.-P. Sun, and F. Nori, Quantum spin squeezing, Phys. Rep. 509, 89 (2011).

[78] A. Sørensen, L.-M. Duan, J. Cirac, and P. Zoller, Manyparticle entanglement with Bose-Einstein condensates, Nature (London) 409, 63 (2001).

[79] P. Ribeiro and T. Prosen, Integrable Quantum Dynamics of Open Collective Spin Models, Phys. Rev. Lett. 122, 010401 (2019).

[80] J. M. Deutsch, Quantum statistical mechanics in a closed system, Phys. Rev. A 43, 2046 (1991).

[81] M. Srednicki, Chaos and quantum thermalization, Phys. Rev. E 50, 888 (1994).

[82] M. Rigol, V. Dunjko, and M. Olshanii, Thermalization and its mechanism for generic isolated quantum systems, Nature (London) 452, 854 (2008).

[83] J. P. Garrahan and I. Lesanovsky, Thermodynamics of Quantum Jump Trajectories, Phys. Rev. Lett. 104, 160601 (2010).

[84] J. P. Garrahan, R. L. Jack, V. Lecomte, E. Pitard, K. van Duijvendijk, and F. van Wijland, Dynamical First-Order Phase Transition in Kinetically Constrained Models of Glasses, Phys. Rev. Lett. 98, 195702 (2007).

[85] F. Carollo, J. P. Garrahan, I. Lesanovsky, and C. Pérez-Espigares, Making rare events typical in Markovian open quantum systems, Phys. Rev. A 98, 010103(R) (2018).

[86] J. P. Garrahan and M. Guţă, Catching and reversing quantum jumps and thermodynamics of quantum trajectories, Phys. Rev. A 98, 052137 (2018).

[87] N. Bartolo, F. Minganti, J. Lolli, and C. Ciuti, Homodyne versus photon-counting quantum trajectories for dissipative Kerr resonators with two-photon driving, Eur. Phys. J. Spec. Top. 226, 2705 (2017).

[88] A. Griessner, A. J. Daley, S. R. Clark, D. Jaksch, and P. Zoller, Dark-State Cooling of Atoms by Superfluid Immersion, Phys. Rev. Lett. 97, 220403 (2006).

[89] A. Aspect, E. Arimondo, R. Kaiser, N. Vansteenkiste, and C. Cohen-Tannoudji, Laser Cooling Below the One-Photon Recoil Energy by Velocity-Selective Coherent Population Trapping, Phys. Rev. Lett. 61, 826 (1988).

[90] S. Haroche and J.-M. Raimond, Exploring the Quantum: Atoms, Cavities, and Photons (Oxford University Press, Oxford, 2006).

[91] H. M. Wiseman and G. J. Milburn, Quantum Measurement and Control (Cambridge University Press, Cambridge, 2010).

[92] S. Gammelmark and K. Mølmer, Fisher Information and the Quantum Cramér-Rao Sensitivity Limit of Continuous Measurements, Phys. Rev. Lett. 112, 170401 (2014).
[93] H. Touchette, The large deviation approach to statistical mechanics, Phys. Rep. 478, 1 (2009).

[94] A. H. Kiilerich and K. Mølmer, Estimation of atomic interaction parameters by photon counting, Phys. Rev. A 89, 052110 (2014).

[95] A. H. Kiilerich and K. Mølmer, Bayesian parameter estimation by continuous homodyne detection, Phys. Rev. A 94, 032103 (2016).

[96] G. W. Gardiner and P. Zoller, Quantum Noise, 2nd ed. (Springer, Berlin, 2000).

[97] E. del Valle, F. P. Laussy, and C. Tejedor, in Proceedings of the International Conference on Transport and Optical Properties of Nanomaterials, Allahbad, 2009, edited by M. R. Singh and R. H. Lipson AIP Conf. Proc. No. 1147 (AIP, Melville, 2009), p. 238.

[98] E. del Valle, A. Gonzalez-Tudela, F. P. Laussy, C. Tejedor, and M. J. Hartmann, Theory of Frequency-Filtered and TimeResolved N-Photon Correlations, Phys. Rev. Lett. 109, 183601 (2012).

[99] A. Ridolfo, E. del Valle, and M. J. Hartmann, Photon correlations from ultrastrong optical nonlinearities, Phys. Rev. A 88, 063812 (2013).

[100] H. Carmichael and D. Walls, Hysteresis in the spectrum for cooperative resonance fluorescence, J. Phys. B 10, L685 (1977).

[101] C. Cohen-Tannoudji and S. Reynaud, Atoms in strong lightfields: Photon antibunching in single atom fluorescence, Philos. Trans. R. Soc. London Ser. A 293, 223 (1979).

[102] C. A. Schrama, G. Nienhuis, H. A. Dijkerman, C. Steijsiger, and H. G. M. Heideman, Intensity correlations between the components of the resonance fluorescence triplet, Phys. Rev. A 45, 8045 (1992).

[103] G. Nienhuis, Spectral correlations in resonance fluorescence, Phys. Rev. A 47, 510 (1993).

[104] A. Ulhaq, S. Weiler, S. M. Ulrich, R. Roßbach, M. Jetter, and P. Michler, Cascaded single-photon emission from the Mollow triplet sidebands of a quantum dot, Nat. Photon. 6, 238 (2012).

[105] B. Silva, C. Sánchez Muñoz, D. Ballarini, A. GonzálezTudela, M. De Giorgi, G. Gigli, K. West, L. Pfeiffer, E. Del Valle, D. Sanvitto et al., The colored Hanbury Brown-Twiss effect, Sci. Rep. 6, 37980 (2016).

[106] M. Peiris, B. Petrak, K. Konthasinghe, Y. Yu, Z. C. Niu, and A. Muller, Two-color photon correlations of the light scattered by a quantum dot, Phys. Rev. B 91, 195125 (2015).

[107] S. Bounouar, M. Strauß, A. Carmele, P. Schnauber, A. Thoma, M. Gschrey, J.-H. Schulze, A. Strittmatter, S. Rodt, A. Knorr, and S. Reitzenstein, Path-Controlled Time Reordering of Paired Photons in a Dressed Three-Level Cascade, Phys. Rev. Lett. 118, 233601 (2017).

[108] H. Strobel, W. Muessel, D. Linnemann, T. Zibold, D. B. Hume, L. Pezzè, A. Smerzi, and M. K. Oberthaler, Fisher information and entanglement of non-Gaussian spin states, Science $\mathbf{3 4 5}$, 424 (2014).

[109] T. Holstein and H. Primakoff, Field dependence of the intrinsic domain magnetization of a ferromagnet, Phys. Rev. 58, 1098 (1940).

[110] M. D. Srinivas and E. B. Davies, Photon counting probabilities in quantum optics, Opt. Acta 28, 981 (1981). 